Pure and Applied Mathematics Quarterly

Volume 3, Number 3

(Special Issue: In honor of

Leon Simon, Part 2 of 2$)$

$689-735,2007$

\title{
Uniqueness of Multiple-spike Solutions via the Method of Moving Planes
}

\author{
Chang-Shou Lin and Juncheng Wei \\ Dedicated to Professor L. Simon on the occasion of his sixtieth birthday
}

\begin{abstract}
We study the uniqueness of multiple-spike solutions for some singularly perturbed Neumann problems in a ball. We completely classify all two-peaked solutions and, except in some degenerate situations, also all three-peaked solutions. Our main idea is using the method of moving planes to show that in the case of two peaks both of them must be located on a line containing the origin and for three peaks all of them must lie in a two-dimensional hyperplane containing the origin. Then we compute the degree of these solutions (restricted in certain symmetry class) and show their uniqueness.
\end{abstract}

\section{INTRODUCTION}

We consider the following singularly perturbed semilinear elliptic problem

$$
\left\{\begin{array}{l}
\epsilon^{2} \Delta u-b u+f(u)=0 \text { in } \Omega, \\
u>0 \text { in } \Omega \text { and } \frac{\partial u}{\partial \nu}=0 \text { on } \partial \Omega,
\end{array}\right.
$$

where $\Omega$ is a bounded domain in $R^{N}$ with smooth boundary $\partial \Omega, \epsilon>0$ is a small constant, $\Delta:=\sum_{j=1}^{N} \frac{\partial^{2}}{\partial x_{j} \partial x_{j}}$ denotes the Laplace operator in $R^{N}, \nu$ stands for the unit outer normal to $\partial \Omega, b>0$ is a positive constant and $f(t)$ is a $C^{1+\sigma}(R) \cap C_{l o c}^{2}(0,+\infty)$ function such that $f(0)=f^{\prime}(0)=0$. Typical examples of the function $-b u+f(u)$ are

$$
-a u+f(u)=-u+u_{+}^{p} \text { with } u_{+}=\max (0, u), b=1,
$$

Received October 21, 2005.

1991 mathematics Subject Classification.Primary 35B40, 35B45; Secondary 35J40.

Keywords and phrases. multiple-spike solutions, uniqueness, method of moving planes, LiapunovSchmidt reduction 


$$
-b u+f(u)=u(u-a)(1-u) \text { with } 0<a<\frac{1}{2}, b=1+a,
$$

where

$$
1<p<\left(\frac{N+2}{N-2}\right)_{+}\left(=\frac{N+2}{N-2} \text { when } N \geq 3 ;=+\infty \text { when } N=1,2\right) .
$$

Equation (1.1) with (1.2) or (1.3) arises in many branches of the applied sciences. For example, it can be viewed as a steady-state equation for the shadow system of the Gierer-Meinhardt system in biological pattern formation ([18], [39], [51]) or of parabolic equations in chemotaxis, population dynamics and phase transitions ([5], [6],[32], [37]).

Associated with (1.1) is the energy functional $J_{\epsilon}$ defined by

$$
J_{\epsilon}[u]:=\int_{\Omega}\left(\frac{\epsilon^{2}}{2}|\nabla u|^{2}+\frac{b}{2} u^{2}-F(u)\right) d x \quad \text { for } u \in H^{1}(\Omega),
$$

where $F(u)=\int_{0}^{u} f(s) d s$.

It is known that any solution $u$ of (1.1) is a critical point of $J_{\epsilon}$ and vice versa. In this paper, we restrict ourselves to families of solutions $\left\{u_{\epsilon}\right\}_{0<\epsilon<\epsilon_{0}}$ of (1.1) with finite energy, i.e.

$$
\epsilon^{-N} J_{\epsilon}\left[u_{\epsilon}\right]<+\infty \quad \text { for } 0<\epsilon<\epsilon_{0} .
$$

It can be proved that for $\epsilon$ sufficiently small, any family of solutions of (1.1) satisfying (1.5) can have at most a finite number of local maximum points (see [34], [48]). Let the local maximum points be $\left\{P_{1}^{\epsilon}, \ldots, P_{K}^{\epsilon}\right\} \subset \bar{\Omega}$. Then one can show that for $\epsilon$ sufficiently small, we have

(S1) $P_{i}^{\epsilon} \neq P_{j}^{\epsilon}$ if $i \neq j$; there exists $\left\{P_{1}^{0}, \ldots, P_{K}^{0}\right\} \in \bar{\Omega}$ such that $P_{j}^{\epsilon} \rightarrow P_{j}^{0} \in \bar{\Omega}$ as $\epsilon \rightarrow 0$, and $u_{\epsilon}$ attains a strict local maximum at $x=P_{j}^{\epsilon}$, for $j=1, \ldots, K$,

(S2) $c_{0}^{-1} \geq u_{\epsilon}\left(P_{j}^{\epsilon}\right) \geq c_{0}>0$ for some constant $c_{0}$ independent of $\epsilon$ and

(S3) $u_{\epsilon}(x) \rightarrow 0$ as $\epsilon \rightarrow 0$ locally uniformly in $\bar{\Omega} \backslash\left\{P_{1}^{0}, \ldots, P_{K}^{0}\right\}$.

Solutions satisfying (S1)-(S3) and (1.5) are called $K$ spike solutions.

In the pioneering papers [31], [32], [34] and [35], Lin, Ni and Takagi established the existence of least-energy solutions and showed that for $\epsilon$ sufficiently small the 
least-energy solution has only one local maximum point $P^{\epsilon}$ with $P^{\epsilon} \in \partial \Omega$. Moreover, $H\left(P^{\epsilon}\right) \rightarrow \max _{P \in \partial \Omega} H(P)$ as $\epsilon \rightarrow 0$, where $H(P)$ is the mean curvature of $\partial \Omega$ at $P$.

Since then many works have been devoted to finding solutions with multiple spikes for the Neumann problem as well as the Dirichlet problem. See [4], [5], [6], [7], [11], [12], [13], [14], [15], [16],[21], [22], [23], [24], [25], [27], [28], [34], [35], [36], [37], [38], [44], [45], [46], [52], [53], and the references therein. (Recent surveys can be found in [39], [54].) It turns out that for the Neumann problem, there are arbitrarily many multiple interior spike solutions. In particular, it was proved in [22] (see also [6], [13]) that given any bounded domain $\Omega$ and a positive integer $K$, there exists an $\epsilon_{K}$ such that for $0<\epsilon<\epsilon_{K}$ problem (1.1) has at least one $K$-interior spike solution with spikes located at $\left\{P_{1}^{\epsilon}, \ldots, P_{K}^{\epsilon}\right\}$. Moreover, as $\epsilon \rightarrow 0$,

$$
\varphi_{K}\left(P_{1}^{\epsilon}, \ldots, P_{K}^{\epsilon}\right) \rightarrow \max _{\left(P_{1}, \ldots, P_{K}\right) \in \Omega^{K}} \varphi_{K}\left(P_{1}, \ldots, P_{K}\right)
$$

where

$$
\varphi_{K}\left(P_{1}, \ldots, P_{K}\right)=\min _{i, j, k, i \neq j}\left(\frac{\left|P_{i}-P_{j}\right|}{2}, d\left(P_{k}, \partial \Omega\right)\right) .
$$

(1.6) shows that if $\left(P_{1}^{\epsilon}, \ldots, P_{K}^{\epsilon}\right) \rightarrow\left(P_{1}^{0}, \ldots, P_{K}^{0}\right)$ as $\epsilon \rightarrow 0$, then $\left(P_{1}^{0}, \ldots, P_{K}^{0}\right)$ attain the sphere packing positions in $\Omega$ :

$$
\varphi_{K}\left(P_{1}^{0}, \ldots, P_{K}^{0}\right)=\max _{\left(P_{1}, \ldots, P_{K}\right) \in \Omega^{K}} \varphi_{K}\left(P_{1}, \ldots, P_{K}\right) .
$$

(Multiple mixed-boundary-interior spike solutions are also obtained in [23].)

In this paper, we consider mainly the case where the domain $\Omega$ is the unit ball $B=\left\{x \in R^{N}|| x \mid<1\right\}$. The results of [6], [13], [14], [22], [23] and [36] show that there can be an arbitrary number of multiple boundary spikes or multiple interior spikes. In general, the limiting positions of the boundary spikes or interior spikes have certain symmetries. As an example, let us consider the interior three spikes constructed in [22] (see Figure 1). The three locations $P_{1}^{0}, P_{2}^{0}, P_{3}^{0}$ form a perfect triangle which is symmetric under rotation by $\frac{2 \pi}{3}$. Moreover, if $N \geq 3$, one can show that $P_{1}^{0}, P_{2}^{0}, P_{3}^{0}$ and the origin must lie in a two dimensional hyperplane. Naturally, one may ask: does the solution also have this partial symmetry? 


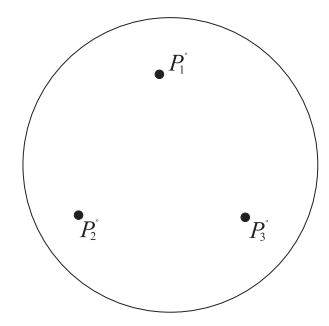

Figure 1

The first result on the partial symmetry of spike solutions of (1.1) is due to Lin and Takagi [30]. (Independently, Grossi [20] obtained the symmetry of the single interior spike solution.) In [30], Lin and Takagi showed that single boundary spike solutions must be axially symmetric, single interior spike solutions must be radially symmetric, and the two boundary spikes $P_{1}^{\epsilon} \in \partial \Omega, P_{2}^{\epsilon} \in \partial \Omega$ must satisfy $P_{1}^{\epsilon}=-P_{2}^{\epsilon}$. By using this information, they showed the uniqueness of the single boundary spike solution and of the two boundary spike solution. We remark that the uniqueness of the single boundary and single interior spike solutions in general domains is studied in [7], [51], [49].

In this paper, we study the partial symmetry for two spikes and three spikes (interior or boundary or mixed). The method of moving planes (MMP) gives us part of the partial symmetry but not the full result. To obtain the full partial symmetry, we have to show the uniqueness of the solutions. To this end, we compute the degree of the solutions.

To illustrate our idea, let us take a look again at the interior three spike solutions (see Figure 1). Suppose we have three interior spikes $P_{1}^{\epsilon}, P_{2}^{\epsilon}, P_{3}^{\epsilon}$ with $P_{j}^{\epsilon} \rightarrow P_{j}^{0}, j=1,2,3$ as $\epsilon \rightarrow 0$. To show the full partial symmetry, we have to show that $P_{j}^{\epsilon}=e^{\sqrt{-1} \frac{2(j-1) \pi}{3}} P_{1}^{\epsilon}, j=$ 2,3 . Note that this is an equation with $3 N$ variables and many symmetries (in other words, degeneracies). To overcome these difficulties, we proceed in two steps:

Step 1. We use MMP to show that $P_{1}^{\epsilon}, P_{2}^{\epsilon}, P_{3}^{\epsilon}$ and the origin must lie in a twodimensional hyperplane and that $u_{\epsilon}$ is axially symmetric with respect to the hyperplane. This reduces our problem to $R^{2}$ with six scalar variables. Now the rotational invariance eliminates one more variable. 
Step 2. We now show that $P_{j}^{\epsilon}=e^{\sqrt{-1} \frac{2(j-1) \pi}{3}} P_{1}^{0}$ when $P_{j}^{\epsilon} \in R^{2}, j=2,3$. To achieve this, we have to compute the degree of $u_{\epsilon}$ restricted to the symmetry class obtained in Step 1. We use the Liapunov-Schmidt reduction method and asymptotic analysis to show that $u_{\epsilon}$ is nondegenerate and that the degree at $u_{\epsilon}$ is exactly $(-1)^{5}$. This proves the uniqueness.

MMP is a powerful method in showing symmetry for Dirichlet problems. For Neumann problems, it has been used recently to show partial symmetry for blow-up and concentration problems ([10], [29], [30]). On the other hand, the method of LiapunovSchmidt reduction has been used in singularly perturbed problems to obtain existence and multiplicity of solutions ([1], [2], [3], [4], [5], [6], [7], [11], [13], [14], [17], [22], [23], [25], [27], [40], [41], [52], [53]). As far as we know, the results of this paper are the first in combining both methods and proving the partial symmetry for three spike solutions. In fact, we are able to completely classify all two spike solutions and, except for some degenerate cases, also all three spike solutions.

\section{Main Results: partial Symmetry and Uniqueness of two AND three} SPIKES

We now state the main theorems of this paper. We always assume that $\Omega=B$ and that $P_{j}^{\epsilon} \in \bar{\Omega}, j=1 \ldots, K$ are the $K$ spikes (boundary or interior). Without loss of generality, we may assume that $b=1$ in (1.1).

First we state the conditions on the function $f(t)$ :

(f1) $f \in C^{1+\sigma}(R) \cap C_{l o c}^{2}(0,+\infty)$ with $0<\sigma \leq 1, f(0)=0, f^{\prime}(0)=0$ and $f(t)=0$ for $t \leq 0$.

(f2) There exists two positive constants $\alpha$ and $\beta$ such that $0<\alpha<\beta,(t-\alpha)(-t+$ $f(t))>0$ for $0<t<\alpha$ or $\alpha<t<\beta$, and $-\frac{1}{2} \beta^{2}+F(\beta)>0$ where $F(t)=\int_{0}^{t} f(s) d s$.

(f3) The problem in the whole space

$$
\left\{\begin{array}{l}
\Delta w-w+f(w)=0, w>0 \text { in } R^{N} \\
w(0)=\max _{y \in R^{N}} w(y), \lim _{|y| \rightarrow+\infty} w(y)=0
\end{array}\right.
$$


has a unique solution $w$, which is nondegenerate, i.e.

$$
\operatorname{Kernel}\left(\Delta-1+f^{\prime}(w)\right)=\operatorname{span}\left\{\frac{\partial w}{\partial y_{1}}, \ldots, \frac{\partial w}{\partial y_{N}}\right\}
$$

By the well-known result of Gidas, $\mathrm{Ni}$ and Nirenberg [19], $w$ is radially symmetric: $w(y)=w(|y|)$ and strictly decreasing: $w^{\prime}(r)<0$ for $r>0, r=|y|$. Moreover, we have the following asymptotic behavior of $w$ :

$$
w(r)=A_{N} r^{-\frac{N-1}{2}} e^{-r}\left(1+O\left(\frac{1}{r}\right)\right), \quad w^{\prime}(r)=-A_{N} r^{-\frac{N-1}{2}} e^{-r}\left(1+O\left(\frac{1}{r}\right)\right),
$$

for $r$ large, where $A_{N}>0$ is a generic constant.

The uniqueness of $w$ is proved in [26] for the case $f(u)=u^{p}$. For a general nonlinearity, see [9]. For $f(u)$ defined by (1.3), the uniqueness of the entire solution was proved by Peletier and Serrin [43].

In what follows we always assume that $f(t)$ satisfies (f1), (f2) and (f3).

Our first theorem concerns the case $K=2$.

Theorem 2.1. Let $K=2$. Then for $\epsilon$ sufficiently small, $P_{1}^{\epsilon}$, the origin, $P_{2}^{\epsilon}$ must lie on a line with 0 between $P_{1}^{\epsilon}$ and $P_{2}^{\epsilon}$. Without loss of generality, we may assume that $P_{1}^{\epsilon}$ and $P_{2}^{\epsilon}$ lie on the $x_{1}$-axis. Then $u_{\epsilon}$ is axially symmetric with respect to $x_{j}, j=2, \ldots, N$.

By Theorem 2.1 and asymptotic analysis (Lemma 5.4 of Section 5 and Lemma 7.1 of Section 7), up to a rotation, there are exactly three possibilities for the limiting positions of the two peaks. These locations are listed below (see Figure 2).

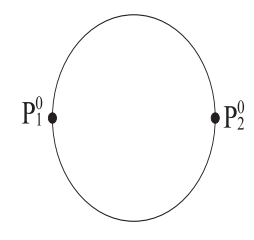

Type 2.I

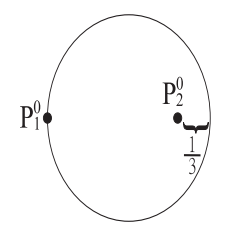

Type 2.II

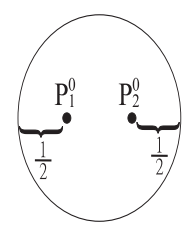

Type 2.III

Figure 2

Our second theorem classifies all two-peaked solutions. 
Theorem 2.2. Let $K=2$. Then for $\epsilon$ sufficiently small, up to a rotation, there are exactly three two-peaked solutions: $u_{\epsilon}^{1}, u_{\epsilon}^{2}, u_{\epsilon}^{3}$. The limiting locations of the three two-peaks are as above (Figure 2).

Moreover, $u_{\epsilon}^{1}, u_{\epsilon}^{3}$ are symmetric with respect to $x_{j}, j=1, \ldots, N, u_{\epsilon}^{2}$ is symmetric with respect to $x_{j}, j=2, \ldots, N$.

We note that the existence of type 2.I solutions was proved in [36] and the existence of type 2.III solutions was proved in [6], [13] and [22]. We remark that the existence and uniqueness of type 2.II solution is new. Combining Theorem 2.1 and Theorem 2.2, we have classified all two-peaked solutions.

Next we consider the three-peak case, which is more complicated.

Our third theorem shows that the three peaks and the origin must lie in a twodimensional hyperplane.

Theorem 2.3. Let $K=3$ and let $P_{1}^{\epsilon}, P_{2}^{\epsilon}, P_{3}^{\epsilon}$ be the three local maximum points of a three-peaked solution $u_{\epsilon}$ of (1.1). Then for $\epsilon$ sufficiently small, $P_{1}^{\epsilon}, P_{2}^{\epsilon}, P_{3}^{\epsilon}$ and the origin must lie in a two-dimensional hyperplane. Without loss of generality, we may assume that the hyperplane is $\Gamma:=\left\{\left(x_{1}, \ldots, x_{N}\right) \mid x_{3}=\ldots=x_{N}=0\right\}$. Then $u_{\epsilon}$ is axially symmetric with respect to $x_{j}, j=3, \ldots, N$. Moreover, the origin must be in the interior of the triangle formed by $P_{1}^{\epsilon} P_{2}^{\epsilon} P_{3}^{\epsilon}$.

Our last theorem concerns the uniqueness of three-peaked solutions. By Theorem 2.3 and some asymptotic analysis (Lemma 5.4 of Section 5 as well as Lemma 7.1 and Lemma 7.2 of Section 7), up to a rotation, there are exactly seven possibilities for the limiting positions of the three peaks. The locations are listed below (see Figure 3). 


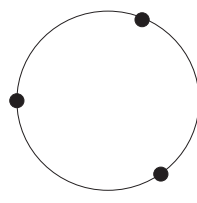

Type 3.I

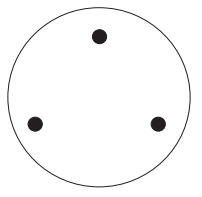

Type 3.IV

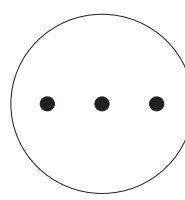

Type 3.VII

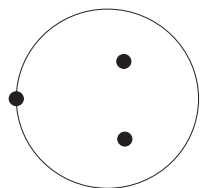

Type 3.II

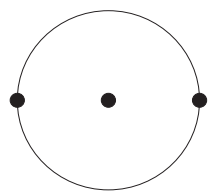

Type 3.V

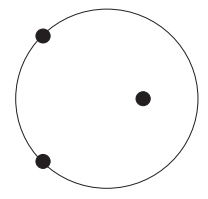

Type 3.III

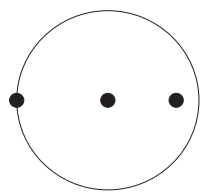

Type 3.VI

Figure 3

We have

Theorem 2.4. Let $K=3$. Then for $\epsilon$ sufficiently small, there are at least seven types of three-peaked solutions. Among them, the first four types are unique. Each of these four types of solution is symmetric in $x_{j}, j=3, \ldots, N$ and inherits the partial symmetry of the locations, e.g., type 3.II, type 3.III solutions are symmetric in $x_{2}$, type 3.I and type $3 . I V$ are symmetric in $x_{j}, j=1, \ldots, N$ and rotationally invariant by $\frac{2 \pi}{3}$.

The other three types of solutions are symmetric with respect to $x_{j}, j=3, \ldots, N$.

The existence of a type 3.I solution was proved in [36] and the existence of a type 3.IV solution was proved in [6], [13] and [22]. The existence of the other five types of solutions is new.

The uniqueness of the other three types 3.V, 3.VI, 3.VII remains open. The main problem is that we can not show that the solutions are symmetric with respect to the $x_{2}$-axis. Once this is shown, it can be proven that they are unique. 
It is natural to ask what happens when $K \geq 4$. We pose the following conjecture at the end:

Conjecture: If the limiting problem (1.8) has a certain partial symmetry, then for $\epsilon$ sufficiently small, the solutions obtained in [22] inherit that partial symmetry.

In this paper, we shall study the uniqueness of type 3.IV solutions of three-peaked solutions in detail, since it is the most complicated and it has the largest number of degrees of freedom. The proof of the uniqueness of the other types will be given in the last section.

The structure of the paper is as follows:

In Section 3, we shall give the proof of Theorems 2.1 and 2.3 by applying the wellknown method of moving planes (MMP) to Neumann problems. This is the MMP part of the paper.

From Section 4 to Section 6, we prove the uniqueness of type 3.IV solutions in the class of partial symmetric functions given by Theorem 2.3. This is the LiapunovSchmidt reduction part of the paper.

In Section 4, we present some preliminaries on the reduction from the infinite dimensional space $H^{1}(\Omega)$ to a finite dimensional problem on the space of spikes. In Section 5 , we classify all types of limiting positions of two or three spikes. In Section 6, we show the uniqueness of the type 3.IV solution by computing its Morse index and degree (restricted to certain symmetric class).

Finally in Section 7, we show how similar ideas can be adopted to prove the uniqueness of other types of solutions. Several technical estimates are contained in Appendices A, B, C and D.

Throughout the paper, we use $C$ to denote various constants independent of $\epsilon$ small. It is always assumed that $\epsilon>0$ is small and $\delta>0$ is a fixed but small constant. 
3. Method of Moving Planes and the Proofs of Theorems 2.1 and 2.3

In this section, we apply the well-known method of moving planes to (1.1). We follow the proofs given in Section 3 of [30], where it is shown that for two boundary spikes $P_{1}^{\epsilon}, P_{2}^{\epsilon}$ it holds that $P_{1}^{\epsilon}=-P_{2}^{\epsilon}$, provided that $\epsilon$ is sufficiently small.

To describe the local structure of spike-layer solutions, we need to introduce a diffeomorphism $z=\Phi_{\epsilon}^{j}(x)$ which is defined in a neighborhood of each concentration point $P_{j}^{\epsilon}$. If $P_{j}^{\epsilon} \in \Omega$, then $\Phi_{\epsilon}^{j}(x)=x$ and $\Omega_{\epsilon, R}^{j}=B_{\epsilon R}\left(P_{j}^{\epsilon}\right)=\left\{x|| x-P_{j}^{\epsilon} \mid \leq \epsilon R\right\}$. If $P_{j}^{\epsilon} \in \partial \Omega$, then $z=\Phi_{\epsilon}^{j}(x)$ maps the boundary portion of $\partial \Omega$ at $P_{j}^{\epsilon}$ to $(0, \ldots, 0,1)$. For details we refer to the reader to [34] and [30]. We also assume that $\Phi_{\epsilon}^{j}$ maps the interior of $\Omega$ to the lower half-space $R_{-}^{N}=\left\{z \mid z_{N}<0\right\}$. Let $B_{R}^{-}:=\left\{z \in R_{-}^{N}|| z \mid<R\right\}$. Set $\Omega_{\epsilon, R}^{j}=\left(\Phi_{\epsilon}^{j}\right)^{-1}\left(B_{\epsilon R}^{-}\right)$.

We state a general result on the asymptotic behavior of $K$-spikes.

Proposition 3.1. Let $\left\{u_{\epsilon}\right\}$ be a family of solutions to (1.1) with $K$ spikes $P_{j}^{\epsilon} \in \bar{\Omega}, j=$ $1, \ldots, K$. Suppose that

$$
\lim _{\epsilon \rightarrow 0} \frac{d\left(P_{j}^{\epsilon}, \partial \Omega\right)}{\epsilon}<+\infty, \quad j=1, \ldots, l
$$

and

$$
\lim _{\epsilon \rightarrow 0} \frac{d\left(P_{j}^{\epsilon}, \partial \Omega\right)}{\epsilon}=+\infty, \quad j=l+1, \ldots, K
$$

Then $P_{j}^{\epsilon} \in \partial \Omega, j=1, \ldots, l$ provided $\epsilon$ sufficiently small. Moreover, for any $\delta>0$, there exists $R=R(\delta)$ and $\epsilon_{1}=\epsilon(\delta)$ such that the following statements hold if $0<\epsilon<\epsilon_{1}$

$$
\quad\left\|u_{\epsilon}(x)-w\left(\Phi_{j}^{\epsilon}(x) / \epsilon\right)\right\|_{C^{2}\left(\bar{\Omega}_{\delta, R}^{j}\right)} \leq \delta
$$

for $j=1, \ldots, K$;

$$
u_{\epsilon}(x) \leq C \delta e^{-\mu \operatorname{dist}\left(x,\left\{P_{1}^{\epsilon}, \ldots, P_{K}^{\epsilon}\right\}\right)} \text { for } x \in \bar{\Omega} \backslash \cup_{j=1}^{K} \Omega_{\epsilon, R}^{j}
$$

where $C$ and $\mu$ are positive constants independent of $\epsilon$.

The proof of Proposition 3.1 is similar to that of Theorem 2.1 of [34]. We omit the details here. 
The main results in this section say that for two peaks, they must both lie on a line containing the origin. For three peaks, they must lie in a two-dimensional hyperplane containing the origin. Moreover, the corresponding solutions must be symmetric with respect to the line or the hyperplane. Since the proof of the two-peaked case is similar to that of [30], we focus our attention on three spikes. Without loss of generality, we assume that the three spikes $P_{1}^{\epsilon}, P_{2}^{\epsilon}, P_{3}^{\epsilon}$ lie in a two-dimensional hyperplane $\left\{\left(x_{1}, \ldots, x_{N}\right) \mid x_{3}=t_{\epsilon, 3}, \ldots, x_{N}=t_{\epsilon, N}\right\}$. We need to show that $t_{\epsilon, j}=0, j=3, \ldots, N$ and $u_{\epsilon}$ is symmetric with respect to $x_{j}, j=3, \ldots, N$, provided that $\epsilon$ is sufficiently small. Without loss of generality, we may assume that $t_{\epsilon}:=t_{\epsilon, N}>0$. Assume also that $\left(P_{1,1}^{\epsilon}\right)^{2}+\left(P_{1,2}^{\epsilon}\right)^{2}=\max _{j=1,2,3}\left(\left(P_{j, 1}^{\epsilon}\right)^{2}+\left(P_{j, 2}^{\epsilon}\right)^{2}\right)$ and that $P_{1,2}^{\epsilon}=0, P_{1,1}^{\epsilon}>0$. Let $\theta_{\epsilon}=\arctan \left(\frac{t_{\epsilon, N}}{P_{1,1}^{\epsilon}}\right)$. Let $\bar{P}_{j}^{\epsilon}=\left(P_{j, 1}^{\epsilon}, 0, \ldots, 0\right)$.

Set $e_{\theta}=(\sin \theta, 0, \ldots, 0,-\cos \theta)$ and $\Pi_{N-1}^{\theta}$ be the $(N-1)$-dimensional hyperplane perpendicular to the vector $e_{\theta}$ and $x^{\theta}$ denotes the reflection of $x$ with respect to $\Pi_{N-1}^{\theta}$. Set

$$
w_{\epsilon}^{\theta}(x)=u_{\epsilon}(x)-u_{\epsilon}\left(x^{\theta}\right) \text { for } x \in \Sigma_{\theta}
$$

where $\Sigma_{\theta}$ is the connected component of $\Omega \backslash \Pi_{N-1}^{\theta}$ containing $P_{1}^{\epsilon}$. Obviously $w_{\epsilon}^{\theta}$ satisfies

$$
\left\{\begin{array}{l}
\epsilon^{2} \Delta w_{\epsilon}^{\theta}+c_{\epsilon}^{\theta}(x) w_{\epsilon}^{\theta}=0 \text { in } \Sigma_{\theta}, \\
\frac{\partial w_{\epsilon}^{\theta}}{\partial \nu}(x)=0 \text { on } \partial \Sigma_{\theta} \backslash \Pi_{N-1}^{\theta}, \\
w_{\epsilon}^{\theta}(x)=0 \text { on } \Sigma_{\theta} \cap \Pi_{N-1}^{\theta},
\end{array}\right.
$$

where

$$
c_{\epsilon}^{\theta}(x)=-1+\frac{f\left(u_{\epsilon}(x)\right)-f\left(u_{\epsilon}\left(x^{\theta}\right)\right)}{u_{\epsilon}(x)-u_{\epsilon}\left(x^{\theta}\right)} .
$$

We prove our claim in a series of three steps.

Step 1: We first prove that

$$
w_{\epsilon}^{0}(x)>0 \text { for } x \in \Sigma_{0}=\left\{x \in \Omega \mid x_{N}>0\right\} .
$$

Note that since $P_{j}^{\epsilon}, j=1,2,3$ are the local maximum points of $u_{\epsilon}$ (and one of them must be a global maximum point), we see that $w_{\epsilon}^{0}\left(P_{j}^{\epsilon}\right)>0$ for some $j$. For contradiction, we assume that the set

$$
E_{\epsilon}:=\left\{x \in \Sigma_{0} \mid w_{\epsilon}^{0}(x)<0\right\}
$$


is non-empty. We break the argument into three cases. (The following argument is for a subsequence of $\epsilon_{i} \rightarrow 0$. For simplicity, we use the same notation $\epsilon$ to denote $\epsilon_{i}$.)

Case 1: $\frac{t_{\epsilon}}{\epsilon} \rightarrow+\infty$ as $\epsilon \rightarrow 0$.

In this case, we see that for arbitrarily large $R>0$, we have $E_{\epsilon} \subset\left(\cup_{j=1}^{3} B_{\epsilon R}\left(P_{j}^{\epsilon}\right)\right)^{c}$. Hence $u_{\epsilon} \leq \delta$ for $x \in E_{\epsilon}$ and $\epsilon$ small. Moreover $w_{\epsilon}^{0}\left(P_{j}^{\epsilon}\right)>0$. Now that

$$
c_{\epsilon}^{\theta}(x) \leq-\frac{1}{2}
$$

for $x \in E_{\epsilon}$. By (3.1), the minimum value of $u_{\epsilon}$, if it is negative, must be obtained on the boundary of $\Sigma_{0}$. Since $\frac{\partial w_{\epsilon}^{\theta}}{\partial \nu}=0$ on $\partial \Omega \cap \Sigma_{0}$ and $w_{\epsilon}^{\theta}=0$ on $\partial \Sigma_{0}$, by the Maximum Principle, $w_{\epsilon}^{0}>0$ and $E_{\epsilon}$ is empty.

Case 2: $C^{-1} \leq \frac{t_{\epsilon}}{\epsilon} \leq C$ for some $C>1$ independent of $\epsilon$.

Let $x_{\epsilon} \in \bar{E}_{\epsilon}$ be such that

$$
w_{\epsilon}^{0}\left(x_{\epsilon}\right)=\inf _{E_{\epsilon}} w_{\epsilon}^{0}(x)<0
$$

Assume for the moment that

$$
\limsup _{\epsilon \rightarrow 0}\left\{\frac{\min _{j=1,2,3}\left|x_{\epsilon}-P_{j}^{\epsilon}\right|}{\epsilon}\right\} \rightarrow+\infty .
$$

Then by Proposition 3.1, $u_{\epsilon}\left(x_{\epsilon}\right) \rightarrow 0$ and $c_{\epsilon}\left(x_{\epsilon}\right)<-\frac{1}{2}<0$ and $0 \leq \epsilon^{2} \Delta w_{\epsilon}^{0}\left(x_{\epsilon}\right)=$ $-c_{\epsilon}^{0}\left(x_{\epsilon}\right) w_{\epsilon}^{0}\left(x_{\epsilon}\right)<0$, a contradiction. Therefore we conclude that

$$
\left|x_{\epsilon}-P_{j}^{\epsilon}\right| \leq R \epsilon
$$

for some $j=1,2,3$. Without loss of generality, we may assume that $\left|x_{\epsilon}-P_{1}^{\epsilon}\right| \leq R \epsilon$. Let

$$
x=\bar{P}_{1}^{\epsilon}+\epsilon y, v_{\epsilon}(y)=u_{\epsilon}(x), x_{\epsilon}=\bar{P}_{1}^{\epsilon}+\epsilon y_{\epsilon} .
$$

Set $y_{\epsilon}=\left(y_{\epsilon}^{\prime}, y_{\epsilon, N}\right)$. Then $y_{\epsilon, N} \geq 0$ and let us assume that $y_{\epsilon, N} \rightarrow y_{0, N} \geq 0, y_{\epsilon}^{\prime} \rightarrow y^{\prime}$. We claim that $y_{0, N}>0$. In fact, by our assumption, $\frac{P_{1, N}^{\epsilon}}{\epsilon} \rightarrow s>0$. Then $v_{\epsilon}(y) \rightarrow w(y)$ in $C_{l o c}^{2}\left(R^{N}\right)$. (If $P_{1}^{\epsilon} \in \partial \Omega$, then we need to extend $v_{\epsilon}$ be reflection. We omit the details.) If $y_{0, N}=0$, then $\frac{\partial w_{\epsilon}^{0}\left(\epsilon y+P_{1}^{\epsilon}\right)}{\partial y_{N}} \rightarrow 2 \frac{\partial w}{\partial y_{N}}\left(y^{\prime}, s\right)<0$ which contradicts to the fact that 
$\nabla w_{\epsilon}^{0}\left(x_{\epsilon}\right)=0$. So $y_{0, N}>0$. In this case, $\frac{\partial v_{\epsilon}}{\partial y_{N}} \rightarrow \frac{\partial w}{\partial y_{N}}>0$ if $y_{N}>0$. Hence $w_{\epsilon}^{0}\left(x_{\epsilon}\right)>0$ for $\epsilon$ small. A contradiction again.

Case 3: $\frac{t_{\epsilon}}{\epsilon}=0$.

In this case, if we write $P_{i}^{\epsilon}=P_{i}^{0}+\epsilon\left(z_{i, \epsilon}^{\prime}, \zeta_{i, \epsilon}\right)$ with $z_{i, \epsilon}^{\prime} \in R^{N-1}, \zeta_{i, \epsilon}>0$, then $z_{i, \epsilon}^{\prime} \rightarrow 0$ and $\zeta_{i, \epsilon} \rightarrow 0$ as $\epsilon \rightarrow 0$. Set $N_{\epsilon}:=\sup _{x \in B_{+}}\left|w_{\epsilon}^{0}(x)\right|$ and let $\bar{x}_{\epsilon} \in \bar{B}_{+}$be such that $\left|w_{\epsilon}^{0}\left(\tilde{x}_{\epsilon}\right)\right|=N_{\epsilon}$. It is easy to see that $\min _{j=1, \ldots, N}\left(\left|\tilde{x}_{\epsilon}-P_{j}^{0}\right|\right) \leq C \epsilon$. Without loss of generality, we may assume that $\left|\tilde{x}_{\epsilon}-P_{1}^{0}\right| \leq C \epsilon$.

Consider the following scaling

$$
\tilde{w}_{\epsilon}^{0}(y):=\frac{1}{N_{\epsilon}} w_{\epsilon}^{0}\left(P_{1}^{0}+\epsilon y\right) .
$$

Let us assume that $\tilde{y}_{\epsilon}:=\frac{\tilde{x}_{\epsilon}-P_{1}^{0}}{\epsilon} \rightarrow \tilde{y}$, where $\tilde{y}=\left(\tilde{y}^{\prime}, \tilde{\eta}\right)$ satisfying $\tilde{\eta} \geq 0$.

Similar to the proof of case 3 of Section 3 of [30], we see that $\tilde{w}_{\epsilon}^{0}(y) \rightarrow \tilde{v}(y)$ in $C_{l o c}^{2}$, where

$$
\tilde{v}(y)=c \frac{\partial w}{\partial y_{N}}(y)
$$

Since $P_{1}^{\epsilon}$ is a local maximum point of $u_{\epsilon}$, we obtain also that

$$
c<0 \text {. }
$$

One can also verify that for $\epsilon$ sufficiently small

$$
C_{0}^{-1} \leq \frac{\zeta_{\epsilon}}{N_{\epsilon}} \leq C_{0}
$$

We now obtain a contradiction with (3.7). In fact, since $\left|\tilde{x}_{\epsilon}-P_{1}^{0}\right| \leq C \epsilon$, we see that $\tilde{w}_{\epsilon}\left(\tilde{y}_{\epsilon}\right)<0$ but $\tilde{w}_{\epsilon}\left(\tilde{y}_{\epsilon}^{\prime}, 0\right)=0$. By the mean value theorem, there exists a $\xi_{\epsilon}>0$ such that

$$
\frac{\partial \tilde{w}_{\epsilon}^{0}}{\partial y_{N}}\left(\tilde{y}_{\epsilon}^{\prime}, \xi_{\epsilon}\right)<0
$$

which implies that as $\epsilon \rightarrow 0$, we have

$$
0 \geq \lim _{\epsilon \rightarrow 0} \frac{\partial \tilde{w}_{\epsilon}^{0}}{\partial y_{N}}\left(\tilde{y}_{\epsilon}^{\prime}, \xi_{\epsilon}\right)=\frac{\partial v}{\partial y_{N}}\left(y_{*}, 0\right)=c \frac{\partial^{2} w}{\partial y_{N}^{2}}\left(y_{*}^{\prime}, 0\right)=c \frac{w^{\prime}\left(\left|y_{*}^{\prime}\right|\right)}{\left|y_{*}^{\prime}\right|}>0
$$

which is a contradiction. 
Consequently, $E_{\epsilon}$ must be empty for $\epsilon$ sufficiently small.

This finishes Step 1.

Step 2. Let

$$
\theta_{0}=\sup \left\{\bar{\theta} \mid w_{\epsilon}^{\theta}>0 \text { for } x \in \Sigma_{\theta} \text { and } 0 \leq \theta \leq \bar{\theta}\right\} .
$$

By the same argument as in Step 1, we see that

$$
\theta_{0} \geq \theta_{\epsilon}
$$

Note that by the definition of $\theta_{0}, w_{\epsilon}^{\theta_{0}} \geq 0$ in $\Sigma_{\theta_{0}}$, and (ii) if $w_{\epsilon}^{\theta_{0}}(x)>0$ for some $x \in \Sigma_{\theta_{0}}$ then $w_{\epsilon}^{\theta_{0}}>0$ in $\Sigma_{\theta_{0}}$ by the maximum principle. Hence we see that $w_{\epsilon}^{\theta_{0}} \equiv 0$ on $\Sigma_{\theta_{0}}$. Since $P_{1}^{\epsilon}$ is a local maximum point, we see that $\theta_{0} \leq \theta_{\epsilon}$. Hence $\theta_{0} \equiv \theta_{\epsilon}$ and $w_{\epsilon}^{\theta_{\epsilon}}(x) \equiv 0$ for $x \in \Sigma_{\theta_{\epsilon}}$. Since $u_{\epsilon}$ has exactly three local maximum points, this implies that $P_{1}^{\epsilon}, P_{2}^{\epsilon}, P_{3}^{\epsilon}$ must lie in the hyperplane $\partial \Sigma_{\theta_{\epsilon}}$. This shows that $P_{j}^{\epsilon}, j=1,2,3$ and the origin lie in a two-dimensional hyperplane.

Step 3: By Step $2, P_{j}^{\epsilon}, j=1,2,3$ and the origin lie in a two-dimensional hyperplane. Without loss of generality, we may assume that the hyperplane is $\Gamma=\left\{x_{3}=\ldots=x_{N}=\right.$ $0\}$. Now we show that

$$
w_{\epsilon}^{0}(x) \equiv 0 \text { on } \Sigma_{0} .
$$

Suppose that there is a sequence $\epsilon_{j} \rightarrow 0$ such that

$$
N_{i}=\sup _{x \in \Sigma_{0}}\left|w_{i}^{0}(x)\right|>0,
$$

where $w_{i}^{0}$ stands for $w_{\epsilon_{i}}^{0}$. Choose an $x_{i} \in \bar{\Sigma}_{0}$ so that $\left|w_{i}^{0}\left(x_{i}\right)\right|=N_{i}$. As in Case 2 above, we can show that

$$
\limsup _{i \rightarrow+\infty} \min _{j=1,2,3}\left(\frac{\left|x_{i}-P_{j}^{\epsilon}\right|}{\epsilon}\right)<+\infty .
$$

Without loss of generality, we may assume that $\frac{\left|x_{i}-P_{1}^{\epsilon}\right|}{\epsilon} \leq C$. As before, let

$$
v_{i}(y)=\frac{1}{N_{i}} w_{i}^{0}\left(P_{1}^{\epsilon}+\epsilon y\right) .
$$

Then, along a subsequence, $\left\{v_{i}\right\}$ converges to $v$ in $C_{l o c}^{2}$, and similar as before

$$
v(y)=c \frac{\partial w}{\partial y_{N}}(y)
$$


for some constant $c \neq 0$. Since $u_{\epsilon}\left(P_{1}^{\epsilon}\right)=0$, we have $\frac{\partial v_{i}}{\partial y_{N}}(0)=0$, so that $\frac{\partial v}{\partial y_{N}}(0)=0$ which implies that $c=0$ since $\frac{\partial^{2} w}{\partial y_{N}^{2}}(0) \neq 0$. A contradiction. Consequently, $w_{\epsilon}^{0}(x) \equiv 0$ on $\Sigma_{0}$, i.e., $u_{\epsilon}\left(x^{\prime}, x_{N}\right)=u_{\epsilon}\left(x^{\prime}, x_{N}\right)$. Similarly, we have $u_{\epsilon}$ is symmetric in $x_{j}, j=$ $3, \ldots, N$.

\section{Preliminaries I: Reduction to Finite-Dimensional Problem}

From this section until Section 6, we shall prove the uniqueness of type 3.IV solutions. (Existence is given in [6], [13] and [22].) Our main idea is to show that type 3.IV solutions are nondegenerate (in some symmetry class) and to compute the Morse index of such solutions. We remark that the uniqueness and Morse index of boundary spikes have been studied in [6] and [49]. The analysis here is more complicated due to the fact that we are dealing with exponentially small orders.

We first introduce a general framework. This framework is a combination of the Liapunov-Schmidt reduction method and the variational principle. The LiapunovSchmidt reduction method has been introduced and used in a lot of papers. See [1], [4], [5], [6], [7], [17], [22], [23], [25], [40], [41], [52], [53] and the references therein. A combination of the Liapunov-Schmidt reduction method and the variational principle was used in [5], [11], [13], [14], [22] and [23]. We shall follow the procedure in [22] which consists of the following steps:

Step 1. Choose good approximate functions.

Recall that $\Omega=B$. Let $w$ be the unique solution of (2.1). We fix a point $P \in \bar{\Omega}$ and introduce the following functions as good approximate functions - the "projection" of $w$ in $H^{1}(\Omega)$. This projection was first introduced in [48] and later studied in [47]. The idea of projecting a function has been used in other problems. See [5], [8], [38], [42], [52], [53] and the references therein. 
We define $w_{\epsilon, P}$ to be the unique solution of

$$
\left\{\begin{array}{l}
\epsilon^{2} \Delta w_{\epsilon, P}-w_{\epsilon, P}+f\left(w\left(\frac{x-P}{\epsilon}\right)\right)=0 \text { in } \Omega \\
w_{\epsilon, P}>0 \text { in } \Omega, \frac{\partial w_{\epsilon, P}}{\partial \nu}=0 \text { on } \partial \Omega
\end{array}\right.
$$

Set

$$
\bar{w}_{\epsilon, P}=w\left(\frac{x-P}{\epsilon}\right), w_{\epsilon, P}=\bar{w}_{\epsilon, P}(x)+\varphi_{\epsilon, P}(x) .
$$

Then $\varphi_{\epsilon, P}$ satisfies

$$
\left\{\begin{array}{l}
\epsilon^{2} \Delta \varphi_{\epsilon, P}-\varphi_{\epsilon, P}=0 \text { in } \Omega \\
\frac{\partial \varphi_{\epsilon, P}}{\partial \nu}=-\frac{\partial}{\partial \nu} \bar{w}_{\epsilon, P} \text { on } \partial \Omega
\end{array}\right.
$$

To study the properties of $\varphi_{\epsilon, P}$, we need to introduce the so-called distance function: let $P \in \Omega$, we define

$$
d_{P}:=d(P, \partial \Omega)=1-|P|
$$

For $P \neq 0$, it is easy to compute that

$$
\begin{gathered}
\nabla_{P} d_{P}=-\frac{P}{|P|} \\
\frac{\partial^{2}}{\partial P_{i} \partial P_{j}} d_{P}=-\frac{1}{|P|}\left(\delta_{i j}-\frac{P_{i} P_{j}}{|P|^{2}}\right)
\end{gathered}
$$

where $P=\left(P_{1}, \ldots, P_{N}\right)$. (Note that $d_{P}$ is not differentiable at $P=0$.)

We state the following useful lemma about the properties of $\varphi_{\epsilon, P}$ and the computations of some integrals. The proof of it is technical and thus delayed to Appendix A.

Lemma 4.1. Let $\Omega=B$ and $P \in \Omega$.

(1) For $\epsilon$ sufficiently small, we have

$$
\varphi_{\epsilon, P}(x)=(1+o(1)) w\left(\frac{x-P}{\epsilon}\right), \text { for } x \in \partial \Omega,
$$

and

$$
-\epsilon \log \varphi_{\epsilon, P}(P) \rightarrow 2 d_{P}, \quad \text { as } \epsilon \rightarrow 0 .
$$

(2) If we further assume that $|P| \geq d_{0}$ for some $d_{0}>0$, then we have

$$
\varphi_{\epsilon, P}(P+\epsilon y)=\varphi_{\epsilon, P}(P)(1+o(1)) e^{-<\nabla d_{P}, y>}, \text { for } P+\epsilon y \in \bar{\Omega},
$$




$$
\varphi_{\epsilon, P}(P)=\left(c_{N}+o(1)\right)\left(d_{P}\left(1-d_{P}\right)\right)^{-\frac{N-1}{2}} \epsilon^{N-1} e^{-2 d_{P} / \epsilon},
$$

where $c_{N}>0$ is a generic constant (depending on $N$ only), and

$$
\begin{gathered}
\int_{\Omega} f^{\prime}\left(\bar{w}_{\epsilon, P}\right) \frac{\partial \bar{w}_{\epsilon, P}}{\partial P_{i}} \varphi_{\epsilon, P}(x) d x \\
=\left(-\gamma_{1}+o(1)\right) \epsilon^{N-1} \varphi_{\epsilon, P}(P)\left(\nabla d_{P}\right)_{i}+O\left(e^{-(2+\sigma) d_{P} / \epsilon}\right)
\end{gathered}
$$

where $\left(\nabla d_{P}\right)_{i}$ denotes the $i$-th component of $\nabla d_{P}$ (which is $-P_{i} /|P|$ in our case) and

$$
\gamma_{1}=\int_{R^{N}} f(w) e^{-y_{1}} d y>0, \sigma=\min (p-1,1) .
$$

(3) For $\epsilon$ sufficiently small and $P_{1}, P_{2} \in \Omega, \frac{\left|P_{1}-P_{2}\right|}{\epsilon} \rightarrow+\infty$, we have

$$
\begin{gathered}
\int_{\Omega} f^{\prime}\left(\bar{w}_{\epsilon, P_{1}}\right) \bar{w}_{\epsilon, P_{2}} \frac{\partial \bar{w}_{\epsilon, P_{1}}}{\partial P_{1, i}} \\
=\epsilon^{N-1}\left(-\gamma_{1}+o(1)\right) w\left(\frac{\left|P_{1}-P_{2}\right|}{\epsilon}\right)\left(\nabla_{P_{1}}\left(\left|P_{1}-P_{2}\right|\right)\right)_{i}+O\left(e^{-(1+\sigma)\left|P_{1}-P_{2}\right| / \epsilon}\right)
\end{gathered}
$$

where $\gamma_{1}$ is given by (4.12).

Step 2. Finite-dimensional reduction.

We now describe the so-called Liapunov-Schmidt finite dimension reduction procedure. Most of the material is from Sections 3,4 and 5 in [22]. See also Sections 4, 5 and 6 in [23].

We first introduce some notations.

We observe that solving (1.1) is equivalent to finding a zero of the following nonlinear equation:

$$
S_{\epsilon}[u]:=\Delta u-u+f\left(u_{+}\right)=0, u \in H_{\nu}^{2}\left(\Omega_{\epsilon}\right)
$$

where

$$
\begin{gathered}
\Omega_{\epsilon}=\{y \mid \epsilon y \in \Omega\} \\
H_{\nu}^{2}\left(\Omega_{\epsilon}\right):=\left\{u \in H^{2}\left(\Omega_{\epsilon}\right) \mid \frac{\partial u}{\partial \nu}=0 \text { on } \partial \Omega_{\epsilon}\right\} .
\end{gathered}
$$

For any $u, v \in H^{1}(\Omega)$, we define the inner product and the norm as follows:

$$
<u, v>_{\epsilon}=\epsilon^{-N} \int_{\Omega}\left(\epsilon^{2} \nabla u \cdot \nabla v+u \cdot v\right), \quad\|u\|_{\epsilon}=<u, v>_{\epsilon}^{\frac{1}{2}} .
$$


We consider $K$-interior spikes. The case of boundary spikes or mixed boundaryinterior spikes will be discussed in Section 7. Fix $\mathbf{P}=\left(P_{1}, \ldots, P_{K}\right) \in \mathbf{\Omega}^{K}$. Let $\varphi_{K}(\mathbf{P})=$ $\varphi_{K}\left(P_{1}, \ldots, P_{K}\right)$ be defined at $(1.7)$. We assume that

$$
\mathbf{P} \in \Lambda_{\delta}=\left\{\mathbf{P} \in \Omega^{K} \mid \varphi_{K}(\mathbf{P}) \geq 2 \delta\right\}
$$

where $\delta$ is a small but fixed positive constant.

To simplify notations, we use the following simplified symbols:

$$
\partial_{j, i}:=\frac{\partial}{\partial P_{j, i}}, w_{\epsilon, \mathbf{P}}=\sum_{j=1}^{K} w_{\epsilon, P_{j}} .
$$

We remark that the variable of $w_{\epsilon, \mathbf{P}}$ is in $\Omega$. Sometimes, we also consider $w_{\epsilon, \mathbf{P}}(\epsilon y)$ for $y \in \Omega_{\epsilon}$. We denote $w_{\epsilon, \mathbf{P}}(\epsilon y)$ as $w_{\epsilon, \mathbf{P}}$.

Now we define the approximate kernel and cokernel respectively as follows:

$$
\begin{aligned}
\mathcal{K}_{\epsilon, \mathbf{P}} & :=\operatorname{span}\left\{\partial_{j, i} w_{\epsilon, \mathbf{P}} \mid j=1, \ldots, K, i=1, \ldots, N\right\} \subset H_{\nu}^{2}\left(\Omega_{\epsilon}\right), \\
\mathcal{C}_{\epsilon, \mathbf{P}} & :=\operatorname{span}\left\{\partial_{j, i} w_{\epsilon, \mathbf{P}} \mid j=1, \ldots, K, i=1, \ldots, N\right\} \subset L^{2}\left(\Omega_{\epsilon}\right) .
\end{aligned}
$$

(Note that $\partial_{j, i} w_{\epsilon, \mathbf{P}} \in H_{\nu}^{2}\left(\Omega_{\epsilon}\right)$ as one can differentiate equation (4.1).)

We also need the following spaces

$$
\begin{aligned}
& \mathcal{K}_{\epsilon, \mathbf{P}}^{\perp}=\left\{u \in H_{\nu}^{2}\left(\Omega_{\epsilon}\right) \mid \int_{\Omega_{\epsilon}} u \partial_{j, i} w_{\epsilon, \mathbf{P}}=0, j=1, \ldots, K, i=1, \ldots, N\right\}, \\
& \mathcal{C}_{\epsilon, \mathbf{P}}^{\perp}=\left\{u \in L^{2}\left(\Omega_{\epsilon}\right) \mid \int_{\Omega_{\epsilon}} u \partial_{j, i} w_{\epsilon, \mathbf{P}}=0, j=1, \ldots, K, i=1, \ldots, N\right\} .
\end{aligned}
$$

Set

$$
\tilde{L}_{\epsilon, \mathbf{P}}(\phi)=\Delta \phi-\phi+f^{\prime}\left(w_{\epsilon, \mathbf{P}}\right) \phi, \quad \mathcal{L}_{\epsilon, \mathbf{P}}=\pi_{\epsilon, \mathbf{P}}^{\perp} \circ \tilde{L}_{\epsilon, \mathbf{P}}
$$

for $\phi \in H_{\nu}^{2}\left(\Omega_{\epsilon}\right)$, where $\pi_{\epsilon, \mathbf{P}}^{\perp}$ is the projection from $L^{2}\left(\Omega_{\epsilon}\right)$ into $\mathcal{C}_{\epsilon, \mathbf{P}}^{\perp}$.

We recall the following result in [47] (see Propositions 3.1 and 3.2 in [47]).

Lemma 4.2. For $\epsilon<<1, \mathcal{L}_{\epsilon, \mathbf{P}}: \mathcal{K}_{\epsilon, \mathbf{P}}^{\perp} \rightarrow \mathcal{C}_{\epsilon, \mathbf{P}}^{\perp}$ is one-to-one and onto. Moreover, the inverse of $\mathcal{L}_{\epsilon, \mathbf{P}}$ exists and bounded (independent of $\epsilon>0$ ).

Next, we have 
Lemma 4.3. For $\epsilon$ sufficiently small, $\mathbf{P} \in \Lambda_{\delta}$, there exists a unique $v_{\epsilon, \mathbf{P}} \in \mathcal{K}_{\epsilon, \mathbf{P}}^{\perp}$ such that

$$
S_{\epsilon}\left(w_{\epsilon, \mathbf{P}}+v_{\epsilon, \mathbf{P}}\right) \in \mathcal{C}_{\epsilon, \mathbf{P}}
$$

Moreover, $v_{\epsilon, \mathbf{P}}$ is $C^{2}$ in $\mathbf{P}$ and

$$
\begin{gathered}
\left\|v_{\epsilon, \mathbf{P}}\right\|_{\epsilon} \leq C e^{-(1+\sigma) \varphi_{K}(\mathbf{P}) / \epsilon} \\
\left\|\partial_{j, i} v_{\epsilon, \mathbf{P}}\right\|_{\epsilon} \leq C \epsilon^{-2} e^{-(1+\sigma) \varphi_{K}(\mathbf{P}) / \epsilon}
\end{gathered}
$$

where $\sigma=\min (1, p-1)$.

Proof: The proof of this Lemma is similar to that of Lemma 2.4 of [51]. For the sake of completeness, we include it in Appendix B.

Step 3. Solve the finite dimensional problem.

Fix any $\mathbf{P} \in \Lambda_{2 \delta}$. Let $v_{\epsilon, \mathbf{P}}$ be the unique solution of (4.22) given by Lemma 4.3. Now we define

$$
\begin{gathered}
M_{\epsilon}(\mathbf{P})=M_{\epsilon}\left(P_{1}, \ldots, P_{K}\right):=\epsilon^{-N} J_{\epsilon}\left[w_{\epsilon, \mathbf{P}}+v_{\epsilon, \mathbf{P}}\right] \\
M_{\epsilon}(\mathbf{P}): \Lambda_{2 \delta} \rightarrow R,
\end{gathered}
$$

where $J_{\epsilon}$ is the energy functional introduced in (1.4) of Section 1.

By Lemma 4.3, $M_{\epsilon}(\mathbf{P}) \in C^{2}\left(\Lambda_{2 \delta}\right)$. Then we have the following reduction theorem.

Lemma 4.4. (Proposition 4.1 of [22]) $u_{\epsilon}=w_{\epsilon, \mathbf{P}^{\epsilon}}+v_{\epsilon, \mathbf{P}^{\epsilon}}, \mathbf{P}^{\epsilon} \in \Lambda_{2 \delta}$ is a critical point of $J_{\epsilon}$ if and only if $P^{\epsilon}$ is a critical point of $M_{\epsilon}(\mathbf{P})$.

Therefore, to prove the existence and uniqueness of solutions of (1.1), we just need to concentrate on the study of critical points of $M_{\epsilon}(\mathbf{P})$, which is a finite-dimensional problem. We shall compute $\nabla M_{\epsilon}(\mathbf{P})$ and $\nabla^{2} M_{\epsilon}(\mathbf{P})$ in the next two sections. 


\section{Preliminaries II: Computations of $\nabla M_{\epsilon}(\mathbf{P})$ and $\nabla^{2} M_{\epsilon}(\mathbf{P})$}

In this section, we first obtain a general formula for the locations of $K$ interior spikes $\left(P_{1}^{\epsilon}, \ldots, P_{K}^{\epsilon}\right)$ and classify all types of limiting locations. Then we compute the (first and second order) derivatives of $M_{\epsilon}(\mathbf{P})$.

The following theorem shows that there will be no spike collapsing to the boundary or with each other.

Lemma 5.1. Let $\left(P_{1}^{\epsilon}, \ldots, P_{K}^{\epsilon}\right)$ be the $K$ local maximum points of a $K$-peaked solution $u_{\epsilon}$ of (1.1). Suppose that $K \leq 3$. Let $P_{j}^{\epsilon} \rightarrow P_{j}^{0}$ for $j=1, \ldots, K$. Then we have

$$
P_{i}^{0} \neq P_{j}^{0}, \text { for } i \neq j,
$$

and if $P_{i}^{0} \in \partial \Omega$, then $P_{i}^{\epsilon} \in \partial \Omega$ for $\epsilon$ sufficiently small.

Lemma 5.1 eliminates the collision of spikes or collision of spikes with the boundary. Note that if the mean curvature of the domain is not constant, one can construct multiple spikes concentrating at one local minimum point of the mean curvature (see [24]). The proof of Lemma 5.1 is technical and thus we delay the proof of it to the appendix C.

By Lemma 5.1, if $P_{j}^{\epsilon}, j=1,2,3$ are three interior spikes, then $\varphi_{K}\left(P_{1}^{\epsilon}, \ldots, P_{K}^{\epsilon}\right) \geq \delta_{0}$ for some $\delta_{0}>0$. Now we choose $\delta=\frac{\delta_{0}}{4}$. By Lemma $4.4, u_{\epsilon}=w_{\epsilon}, \mathbf{P}^{\epsilon}+v_{\epsilon, \mathbf{P}^{\epsilon}}$ is a solution with three interior spikes if and only if $\mathbf{P}^{\epsilon}$ is a critical point of $M_{\epsilon}$, since $\mathbf{P}^{\epsilon} \in \Lambda_{2 \delta}$.

The asymptotic expansion of $M_{\epsilon}(\mathbf{P})$ in $\Lambda_{\delta}$ is given in Proposition 4.1 of [22].

Lemma 5.2. (Proposition 4.1 of $[22]$.) For $\epsilon$ sufficiently small and $\mathbf{P} \in \Lambda_{\delta}$, we have

$$
M_{\epsilon}(\mathbf{P})=K I(w)-\frac{1}{2}\left(\gamma_{1}+o(1)\right)\left(\sum_{i=1}^{K} \varphi_{\epsilon, P_{i}}\left(P_{i}\right)\right)-\left(\gamma_{1}+o(1)\right) \sum_{k \neq l} w\left(\left|P_{k}-P_{l}\right| / \epsilon\right)
$$

where

$$
I(w)=\frac{1}{2} \int_{R^{N}}|\nabla w|^{2}+\frac{1}{2} \int_{R^{N}} w^{2}-\int_{R^{N}} F(w)
$$

and $\gamma_{1}$ is given by (4.12). 
We now show that the asymptotic expansion in (5.2) holds true in $C^{2}$ sense. Set

$$
\tilde{M}_{\epsilon}(\mathbf{P}):=-\frac{\gamma_{1}}{2} \sum_{j=1}^{K} \varphi_{\epsilon, P_{j}^{\epsilon}}\left(P_{j}^{\epsilon}\right)-\gamma_{1} \sum_{k \neq l} w\left(\left|P_{k}-P_{l}\right| / \epsilon\right) .
$$

By (4.10) of Lemma 4.1 and (2.3), we see that if $\left|P_{j}^{\epsilon}\right| \geq \frac{1}{10}, j=1, \ldots, K$, then we have

$$
\begin{gathered}
\tilde{M}_{\epsilon}(\mathbf{P}):=-\frac{c_{N}\left(\gamma_{1}+o(1)\right)}{2} \epsilon^{\frac{N-1}{2}} \sum_{j=1}^{N} c\left(P_{j}^{\epsilon}\right) e^{-2 d_{P_{j}^{\epsilon}} / \epsilon} \\
-A_{N}\left(\gamma_{1}+o(1)\right) \epsilon^{\frac{N-1}{2}} \sum_{k \neq l}\left(\left|P_{k}-P_{l}\right|\right)^{-\frac{N-1}{2}} e^{-\left|P_{k}-P_{l}\right| / \epsilon},
\end{gathered}
$$

where $c_{N}$ is given in (4.10) of Lemma $4.1, A_{N}>0$ is given by (2.3), and

$$
c(P)=\left(d_{P}\left(1-d_{P}\right)\right)^{-\frac{N-1}{2}} .
$$

The following lemma is our key estimate.

Lemma 5.3. Suppose that $\mathbf{P}^{\epsilon} \in \Lambda_{\delta}$ and $\epsilon$ is sufficiently small.

(1) If $\left|P_{j}^{\epsilon}\right| \geq d_{0}$ for some $j$ and $d_{0}>0$, then we have

$$
\partial_{j, i} M_{\epsilon}(\mathbf{P})=\partial_{j, i} \tilde{M}_{\epsilon}(\mathbf{P})+O\left(\tilde{M}_{\epsilon}(\mathbf{P})\right), i=1, \ldots, N .
$$

(2). If $\left|P_{j}^{\epsilon}\right| \leq \frac{1}{10}$ for some $j$, then we have

$$
\partial_{j, i} M_{\epsilon}(\mathbf{P})=-\left(\gamma_{1}+o(1)\right) \sum_{k \neq j} \partial_{j, i}\left(w\left(\left|P_{k}-P_{j}\right| / \epsilon\right)\right)+O\left(\tilde{M}_{\epsilon}(\mathbf{P})\right), i=1, \ldots, N .
$$

(3) Suppose that $\mathbf{P}^{\epsilon}$ is a critical point of $M_{\epsilon}(\mathbf{P})$ such that $\left|P_{j}^{\epsilon}\right| \geq d_{0}, j=1, \ldots, N$ for some $d_{0}>0$. Then we have

$$
\left.\partial_{l, m} \partial_{j, i} M_{\epsilon}(\mathbf{P})\right|_{\mathbf{P}=\mathbf{P}^{\epsilon}}=\left.\partial_{l, m} \partial_{j, i} \tilde{M}_{\epsilon}(\mathbf{P})\right|_{\mathbf{P}=\mathbf{P}^{\epsilon}}+O\left(\epsilon^{-1} \tilde{M}_{\epsilon}\left(\mathbf{P}^{\epsilon}\right)\right), l=1, \ldots, K, m=1, \ldots, N .
$$

More precisely, we have

$$
\begin{gathered}
\left.\partial_{l, m} \partial_{j, i} M_{\epsilon}(\mathbf{P})\right|_{\mathbf{P}=\mathbf{P}^{\epsilon}} \\
=\epsilon^{N-2}\left(\gamma_{1}+o(1)\right) w\left(\left|P_{j}^{\epsilon}-P_{l}^{\epsilon}\right| / \epsilon\right) e_{j l, m}^{\epsilon} e_{j l, i}^{\epsilon}\left(1-\delta_{j l}\right) \\
-\epsilon^{N-2}\left(\gamma_{1}+o(1)\right) \varphi_{\epsilon, P_{j}^{\epsilon}}\left(P_{j}^{\epsilon}\right) e_{j, i}^{\epsilon} e_{l, m}^{\epsilon} \delta_{j l} \\
-\epsilon^{N-2}\left(\gamma_{1}+o(1)\right) \sum_{k \neq j} w\left(\left|P_{j}^{\epsilon}-P_{k}^{\epsilon}\right| / \epsilon\right) e_{j k, i}^{\epsilon} e_{j k, m}^{\epsilon} \delta_{j l},
\end{gathered}
$$


where

$$
e_{j}^{\epsilon}=\frac{P_{j}^{\epsilon}}{\left|P_{j}^{\epsilon}\right|}, e_{j k}^{\epsilon}=\frac{P_{j}^{\epsilon}-P_{k}^{\epsilon}}{\left|P_{j}^{\epsilon}-P_{k}^{\epsilon}\right|}, j \neq k,
$$

and $e_{j, i}^{\epsilon}$ and $e_{j k, i}^{\epsilon}$ denote the $i-$ th component of the vectors $e_{j}^{\epsilon}$ and $e_{j k}^{\epsilon}$, respectively.

Remark: The reason that we have to consider cases when $P_{j}^{\epsilon}$ is close to the origin is that the function $d_{P}$ is not differentiable at the origin. However if we know the rate of $P_{j}^{\epsilon}$ approaching the origin, we may still be able to compute the derivatives (see [51]). This is a delicate issue that needs further investigation. For the purpose of this paper, Lemma 5.3 is good enough.

The proof of Lemma 5.3 is very technical and we will present it in Appendix D.

Let $\mathbf{P}^{\epsilon}=\left(P_{1}^{\epsilon}, \ldots, P_{K}^{\epsilon}\right)$ be a critical point of $M_{\epsilon}(\mathbf{P})$. Namely, we have

$$
\left.\partial_{j, i} M_{\epsilon}(\mathbf{P})\right|_{\mathbf{P}=\mathbf{P}^{\epsilon}}=0, j=1, \ldots, K, i=1, \ldots, N .
$$

(5.10) shows that if $\mathbf{P}^{\epsilon}$ is a critical point of $M_{\epsilon}$ and $\left|P_{j}^{\epsilon}\right| \geq \frac{1}{10}, j=1, \ldots, K$, then we have

$$
\begin{gathered}
\left(\gamma_{1}+o(1)\right) c\left(P_{j}^{\epsilon}\right) e^{-2 d_{P_{j}^{\epsilon}} / \epsilon}\left(\nabla_{P_{j}^{\epsilon}} d_{P_{j}^{\epsilon}}\right)_{i} \\
+\left(\gamma_{1}+o(1)\right) \sum_{l \neq j}\left(\left|P_{l}^{\epsilon}-P_{j}^{\epsilon}\right|\right)^{-\frac{N-1}{2}} e^{-\left|P_{l}^{\epsilon}-P_{j}^{\epsilon}\right| / \epsilon}\left(\nabla_{P_{j}^{\epsilon}}\left|P_{j}^{\epsilon}-P_{l}^{\epsilon}\right|\right)_{i} \\
+O\left(\epsilon\left(\sum_{j=1}^{K} e^{-2 d_{P_{j}^{\epsilon}} / \epsilon}+\sum_{l \neq k} e^{-\left|P_{l}^{\epsilon}-P_{k}^{\epsilon}\right| / \epsilon}\right)\right)=0, j=1, \ldots, K, i=1, \ldots, N .
\end{gathered}
$$

(5.8) and (5.13) enable us to classify all types of locations for the limiting positions of interior two or interior three peaks. Moreover, it also gives us the estimate on the speed of $\mathbf{P}^{\epsilon}$ approaching these limiting positions (Lemma 6.1 in the next section).

Lemma 5.4. Let $K=2$ or 3 and $\tilde{P}_{j}^{\epsilon} \in \Omega, j=1, \ldots, K$ be the $K$ local maximum points of $u_{\epsilon}$. Suppose that $\tilde{P}_{j}^{\epsilon} \rightarrow P_{j}^{0}, j=1, \ldots, K$, then up to a rotation, $\mathbf{P}^{0}=\left(P_{1}^{0}, \ldots, P_{K}^{0}\right)$ must belong to type 2.III (for $K=2$, Figure 2) and either type 3.IV or type 3.VII (for $K=3$, Figure 3). 
Proof: Let $u_{\epsilon}$ be a solution of (1.1) with two interior spikes or three interior spikes. By Lemma 4.4, we have $u_{\epsilon}=w_{\epsilon} \mathbf{P}^{\epsilon}+v_{\epsilon, \mathbf{P}^{\epsilon}}$, where $\mathbf{P}^{\epsilon} \in \Lambda_{\delta}$. Let $\tilde{P}_{j}^{\epsilon}, j=1,2, \ldots, K$ be the $K$ local maximum points of $u_{\epsilon}$. Then, up to a rotation, we have $\tilde{P}_{j}^{\epsilon}-P_{j}^{\epsilon}=o(1), j=$ $1, \ldots, K$. By Lemma 5.1, $P_{j}^{0} \neq P_{i}^{0}$ for $i \neq j$.

Let us consider $K=2$ first. From (5.8), it is easy to see that both $\left|P_{1}^{\epsilon}\right|$ and $\left|P_{2}^{\epsilon}\right|$ must be larger than $\frac{1}{10}$ (as otherwise, (5.8) is not balanced). Thus we may assume that $\left|P_{1}^{\epsilon}\right| \geq \frac{1}{10},\left|P_{2}^{\epsilon}\right| \geq \frac{1}{10}$.

Since both $\tilde{P}_{1}^{\epsilon}$ and $\tilde{P}_{2}^{\epsilon}$ are in the interior, (5.13) implies that we must have

$$
\left|P_{1}^{\epsilon}-P_{2}^{\epsilon}\right|=2 d_{P_{1}^{\epsilon}}+o(1)=2 d_{P_{2}^{\epsilon}}+o(1)
$$

and that $P_{1}^{0}$ and $P_{2}^{0}$ must be anti-pole, i.e. $P_{1}^{0}=-P_{2}^{0}$. This shows that the limiting positions must be type 2.III.

For $K=3$, we proceed similarly. By Lemma 4.1, $P_{j}^{\epsilon} \rightarrow P_{j}^{0}, j=1,2,3$, where $P_{j}^{0} \in \Omega, P_{k}^{0} \neq P_{l}^{0}$ if $k \neq l$. We consider two cases.

Case 1. Suppose one of the points $P_{j}^{0}, j=1,2,3$ is the origin, say $P_{2}^{0}=0$. Then we consider (5.13) at $j=1$ and conclude that $2 d_{P_{1}^{0}}=\left|P_{1}^{0}-P_{2}^{0}\right|$. Similarly we have $2 d_{P_{2}^{0}}=\left|P_{1}^{0}-P_{2}^{0}\right|$. From equation (5.8) at $j=2$, we see that

$$
P_{1}^{0}+P_{2}^{0}=0
$$

which shows that $\left(P_{1}^{0}, P_{2}^{0}, P_{3}^{0}\right)$ is type 3 .VII.

Case 2. Suppose $P_{j}^{0} \neq 0, j=1,2,3$. Then $\left|P_{j}^{\epsilon}\right| \geq d_{0}$ for $j=1,2,3$ and some $d_{0}>0$. We use (5.13) at $j=1,2,3$ to conclude that $\left(P_{1}^{0}, P_{2}^{0}, P_{3}^{0}\right)$ must be type $3 . \mathrm{IV}$ (see the proof of Lemma 6.1 in the next section).

\section{UNIQUENESS OF THE TYPE 3.IV SOLUTIONS}

In this section, we prove the uniqueness of the type 3.IV solution for $\epsilon$ sufficiently small. Let $P_{1}^{0}, P_{2}^{0}, P_{3}^{0}$ be the limiting positions as shown in Figure 3 and let $u_{\epsilon}$ be a three-peaked solution whose local maximum points are $\tilde{P}_{j}^{\epsilon}, j=1,2,3$. By MMP 
(Section 3 ), the solution $u_{\epsilon}$ is symmetric in $x_{j}, j=3, \ldots, N$. Let

$$
H_{\nu, s}^{2}\left(\Omega_{\epsilon}\right)=\left\{u \in H_{\nu}^{2}\left(\Omega_{\epsilon}\right) \mid u \text { is symmetric with respect to } x_{j}, j=3, \ldots, N\right\} .
$$

Consider the following minimization problem

$$
\min _{P_{j} \in \hat{\Lambda}_{2 \delta}}\left\|u_{\epsilon}-\sum_{j=1}^{3} w_{\epsilon, P_{j}}\right\|_{L^{2}\left(\Omega_{\epsilon}\right)}
$$

where

$$
\hat{\Lambda}_{2 \delta}=\left\{\left(P_{1}, P_{2}, P_{3}\right)|| P_{j}-P_{j}^{0} \mid \leq 2 \delta, P_{j, i}=0, j=1,2,3, i \geq 3\right\} .
$$

It is easy to see that (6.2) can be attained and thus we have

$$
u_{\epsilon}=w_{\epsilon, \mathbf{P}^{\epsilon}}+\phi_{\epsilon}
$$

where $\mathbf{P}^{\epsilon} \in \hat{\Lambda}_{\delta}, \phi_{\epsilon} \in H_{\nu, s}^{2}(\Omega)$. Moreover, $\phi_{\epsilon} \in \mathcal{K}_{\epsilon, \mathbf{P}^{\epsilon}}^{\perp}$. Since

$$
S\left[w_{\epsilon, \mathbf{P}^{\epsilon}}+\phi_{\epsilon}\right]=0 \in \mathcal{C}_{\epsilon, \mathbf{P}^{\epsilon}}, \phi_{\epsilon} \in \mathcal{K}_{\epsilon, \mathbf{P}^{\epsilon}}^{\perp},
$$

by Lemma 4.3 , we see that

$$
\phi_{\epsilon}=v_{\epsilon, \mathbf{P}^{\epsilon}}
$$

where $v_{\epsilon, \mathbf{P}^{\epsilon}}$ is defined by Lemma 4.3 . (Note that $P_{j}^{\epsilon}$ may not be a local maximum point of $u_{\epsilon}$. But it is easy to show that up to a permutation, $P_{j}^{\epsilon}=\tilde{P}_{j}^{\epsilon}+o(1), j=1,2,3$.)

For $\mathbf{P} \in \hat{\Lambda}_{\delta}$, we may define $\hat{P}_{j}=\left(P_{j, 1}, P_{j, 2}\right), \hat{\mathbf{P}}=\left(\hat{P}_{1}, \hat{P}_{2}, \hat{P}_{3}\right)$ and

$$
\hat{M}_{\epsilon}(\hat{\mathbf{P}})=M_{\epsilon}(\mathbf{P}) \text {. }
$$

Similar to Lemma 4.4, we have that $\hat{\mathbf{P}}^{\epsilon}$ is a critical point of $\hat{M}_{\epsilon}(\hat{\mathbf{P}})$ if and only if $u_{\epsilon}=w_{\epsilon, \mathbf{P}^{\epsilon}}+v_{\epsilon, \mathbf{P}^{\epsilon}}$ is a critical point of $J_{\epsilon}$.

To avoid clumsy notation, we drop the hat now. Thus our problem is reduced to a six-dimensional problem. Moreover, by rotation, we may fix $P_{11}=0$. Let $\mathbf{p}=$ $\left(P_{12}, P_{21}, P_{22}, P_{31}, P_{32}\right)$. Then if $\mathbf{P}^{\epsilon}$ is a critical point, the corresponding $\mathbf{p}^{\epsilon}$ is also a critical point of $M_{\epsilon}(\mathbf{P})$. Thus all we need to prove is the uniqueness of the critical point of $M_{\epsilon}(\mathbf{P})$ for $\mathbf{P}$ in the set

$$
\omega=\left\{\left(P_{12}, P_{21}, P_{22}, P_{31}, P_{32}\right) \mid \mathbf{P} \in \hat{\Lambda}_{\delta}, P_{11}=0\right\},
$$

which is a five-dimensional problem. 
We begin with the following lemma which computes the speed of $\mathbf{P}^{\epsilon}$ approaching $\mathbf{P}^{0}$. This kind of estimate is needed for the proof of uniqueness of spikes. See [7] and [49].

Lemma 6.1. Let $P_{j}^{\epsilon}, j=1,2,3$ be as above. Then there exists a unique vector $\vec{a} \in R^{2}$ such that

$$
P_{j}^{\epsilon}=P_{1}^{0}+\epsilon e^{\sqrt{-1} \frac{2(j-1) \pi}{3}} \vec{a}+o(\epsilon), j=1,2,3
$$

where $\vec{a}=\left(0, a_{2}\right)$ for some fixed number $a_{2}$.

Proof: Our main tool is equation (5.13).

Adding all the three equations in (5.13), we obtain that

$$
\sum_{j=1}^{3} e^{-\left|P_{j}^{\epsilon}\right| / \epsilon} \frac{P_{j}^{\epsilon}}{\left|P_{j}^{\epsilon}\right|}+o\left(\sum_{j=1}^{3} e^{2\left|P_{j}^{\epsilon}\right| / \epsilon}\right)=0
$$

Since

and

$$
\frac{P_{1}^{\epsilon}}{\left|P_{1}^{\epsilon}\right|}=\vec{e}_{2}=(0,1)
$$

we deduce from (5.13) that

$$
\frac{P_{j}^{\epsilon}}{\left|P_{j}^{\epsilon}\right|}=e^{\sqrt{-1} \frac{2(j-1) \pi}{3}} \vec{e}_{2}+o(1), \quad j=2,3,
$$

$$
\left|P_{j}^{\epsilon}\right|=\left|P_{1}^{\epsilon}\right|+o(\epsilon), d_{P_{j}^{\epsilon}}=d_{P_{1}^{\epsilon}}+o(\epsilon), \quad j=2,3 .
$$

Next we examine equation (5.13) at $j=1$. We have

$$
\frac{P_{1}^{0}}{\left|P_{1}^{0}\right|}+a_{1}^{0} e^{\left(2 d_{P_{1}^{\epsilon}}-\left|P_{2}^{\epsilon}-P_{1}^{\epsilon}\right|\right) / \epsilon} \frac{P_{2}^{0}-P_{1}^{0}}{\left|P_{2}^{0}-P_{1}^{0}\right|}+a_{1}^{0} e^{\left(2 d_{\left.P_{1}^{\epsilon}-\left|P_{2}^{\epsilon}-P_{1}^{\epsilon}\right|\right) / \epsilon}\right.} \frac{P_{3}^{0}-P_{1}^{0}}{\left|P_{3}^{0}-P_{1}^{0}\right|}=o(1)
$$

where $a_{1}^{0}$ is a generic constant. Since the following decomposition is unique,

we see that

$$
\frac{P_{1}^{0}}{\left|P_{1}^{0}\right|}=-\frac{P_{2}^{0}-P_{1}^{0}}{\left|P_{2}^{0}-P_{1}^{0}\right|}-\frac{P_{3}^{0}-P_{1}^{0}}{\left|P_{3}^{0}-P_{1}^{0}\right|}
$$

$$
a_{1}^{0} e^{\left(2 d_{P_{1}^{\epsilon}}-\left|P_{2}^{\epsilon}-P_{1}^{\epsilon}\right|\right) / \epsilon}=1+o(1), a_{1}^{0} e^{\left(2 d_{P_{1}^{\epsilon}}-\left|P_{3}^{\epsilon}-P_{1}^{\epsilon}\right|\right) / \epsilon}=1+o(1),
$$

which implies that

$$
\left|P_{2}^{\epsilon}-P_{1}^{\epsilon}\right|=2 d_{P_{1}^{\epsilon}}+\epsilon a_{0}+o(\epsilon),\left|P_{3}^{\epsilon}-P_{1}^{\epsilon}\right|=2 d_{P_{1}^{\epsilon}}+\epsilon a_{0}+o(\epsilon)
$$


where $a_{0}$ is a generic constant. Similarly, we have

$$
\left|P_{i}^{\epsilon}-P_{j}^{\epsilon}\right|=2 d_{P_{i}^{\epsilon}}+\epsilon a_{0}+o(\epsilon), \quad i \neq j, \quad i, j=1,2,3 .
$$

From (6.9) and (6.11), we see that

$$
P_{j}^{\epsilon}=e^{\sqrt{-1} \frac{2(j-1) \pi}{3}} P_{1}^{\epsilon}+o(\epsilon), \quad j=2,3 .
$$

Substituting (6.12) into (6.10), we see that

$$
P_{1}^{\epsilon}=P_{1}^{0}+\epsilon \vec{a}+o(\epsilon)
$$

for some unique $\vec{a}$. (Note that $\vec{a}=a_{2} \vec{e}_{2}$ for some $a_{2}$.) By (6.12), (6.7) holds.

By Lemma 6.1, any critical point $\mathbf{P}^{\epsilon}$ of $M_{\epsilon}(\mathbf{P})$ in $B_{\delta}\left(\mathbf{P}^{0}\right)$ must satisfy $\mathbf{P}^{\epsilon}=\mathbf{P}^{0}+$ $\epsilon \mathbf{a}+o(\epsilon)$ for some fixed $\mathbf{a}$. Let $\mathbf{Q}^{\epsilon}=\mathbf{P}^{0}+\epsilon \mathbf{a}$.

Our next lemma shows that every critical point $\mathbf{P}^{\epsilon}$ must be nondegenerate.

Lemma 6.2. Let $\mathbf{P}^{\epsilon} \in B_{\delta \epsilon}\left(\mathbf{Q}^{\epsilon}\right)$ be a critical point of $M_{\epsilon}(\mathbf{P})$. Then for $\epsilon$ sufficiently small, we have

$$
\left.\sum_{j, l, m, i} \partial_{l, m} \partial_{j, i} M_{\epsilon}(\mathbf{P})\right|_{\mathbf{P}=\mathbf{P}^{\epsilon}} \eta_{l, m} \eta_{j, i} \geq C \epsilon^{\frac{3 N-5}{2}} e^{-2 \varphi_{K}\left(\mathbf{P}^{\epsilon}\right) / \epsilon}|\eta|^{2}
$$

where $C$ is independent of $\epsilon, \eta=\left(\eta_{11}, \eta_{12}, \eta_{21}, \eta_{22}, \eta_{31}, \eta_{32}\right) \in R^{6}, \eta_{11}=0$ and $|\eta|^{2}=$ $\sum_{i, j} \eta_{i j}^{2}$

Proof: We have by Lemma 5.3 (2),

$$
\begin{gathered}
\left.\sum_{j, l, m, i} \partial_{l, m} \partial_{j, i} M_{\epsilon}(\mathbf{P})\right|_{\mathbf{P}=\mathbf{P}^{\epsilon}} \eta_{l, m} \eta_{j, i} \\
=\left(\gamma_{1}+o(1)\right) \epsilon^{N-2} \sum_{j} \varphi_{\epsilon, P_{j}^{\epsilon}}\left(P_{j}^{\epsilon}\right) \sum_{l}<e_{l}^{\epsilon}, \eta_{l}>^{2} \\
+\left(\gamma_{1}+o(1)\right) \epsilon^{N-2} w\left(\left|P_{1}^{\epsilon}-P_{2}^{\epsilon}\right| / \epsilon\right)(1+o(1)) \sum_{j \neq l}<e_{j l}^{\epsilon}, \eta_{j}-\eta_{l}>^{2} .
\end{gathered}
$$

Since $\mathbf{P}^{\epsilon} \in B_{\delta \epsilon}\left(\mathbf{Q}^{\epsilon}\right),\left|P_{i}^{\epsilon}-P_{j}^{\epsilon}\right|=\left|P_{1}^{\epsilon}-P_{2}^{\epsilon}\right|+o(\epsilon)$ for $i \neq j$ and $d_{P_{j}^{\epsilon}}=d_{P_{1}^{\epsilon}}+o(\epsilon)$. Hence

$$
\varphi_{\epsilon, P_{j}^{\epsilon}}\left(P_{j}^{\epsilon}\right) \sim w\left(\left|P_{1}^{\epsilon}-P_{2}^{\epsilon}\right|\right), \quad j=1,2,3 .
$$


(6.15) shows that

$$
\begin{gathered}
\left.\sum_{j, l, m, i} \partial_{l, m} \partial_{j, i} M_{\epsilon}(\mathbf{P})\right|_{\mathbf{P}=\mathbf{P}^{\epsilon}} \eta_{l, m} \eta_{j, i} \\
\geq C \epsilon^{N-2} e^{-2 \varphi_{K}\left(\mathbf{P}^{\epsilon}\right) / \epsilon}\left(\sum_{l}<e_{l}^{\epsilon}, \eta_{l}>^{2}+\sum_{j \neq l}<e_{j l}^{\epsilon}, \eta_{j}-\eta_{l}>^{2}\right)
\end{gathered}
$$

for some $C>0$ independent of $\epsilon$.

We now show that

$$
\left.\sum_{j, l, m, i} \partial_{l, m} \partial_{j, i} M_{\epsilon}(\mathbf{P})\right|_{\mathbf{P}=\mathbf{P}^{\epsilon}} \eta_{l, m} \eta_{j, i} \geq C \epsilon^{N-2} e^{-2 \varphi_{K}\left(\mathbf{P}^{\epsilon}\right) / \epsilon}|\eta|^{2}
$$

where $C$ is independent of $\epsilon$ and $|\eta|^{2}=\sum_{i, j} \eta_{i j}^{2}$.

To this end, it is enough to show that

$$
\sum_{l}<e_{l}^{\epsilon}, \eta_{l}>^{2}+\sum_{j \neq l}<e_{j l}^{\epsilon}, \eta_{j}-\eta_{l}>^{2} \geq C|\eta|^{2}
$$

if $\eta_{11}=0$. In fact, the left hand side of (6.17) is equal to 0 if and only if

$$
<e_{l}^{\epsilon}, \eta_{l}>=0,<e_{j l}^{\epsilon}, \eta_{l}-\eta_{j}>=0 .
$$

For $j=1,<e_{1}^{\epsilon}, \eta_{1}>=0$, then $\eta_{12}=0$. Hence $\eta_{1}=0$, which implies that $<e_{12}^{\epsilon}, \eta_{2}>=<$ $e_{2}^{\epsilon}, \eta_{2}>=0$. Hence $\eta_{2}=0$. Similarly, we have $\eta_{3}=0$. Thus (6.17) holds true.

(6.14) shows that the matrix $\left(\left.\partial_{l, m} \partial_{j, i} M_{\epsilon}(\mathbf{P})\right|_{\mathbf{P}=\mathbf{P}_{\epsilon}}\right)$ is negatively definite if we restrict to the space $\left\{\eta_{11}=0\right\}$. Thus the Morse index is 5 .

Finally we have

Lemma 6.3. For $\delta>0$ small, there exists a unique critical point of $M_{\epsilon}(\mathbf{P})$ over $B_{\delta \epsilon}\left(\mathbf{Q}^{\epsilon}\right)$.

\section{Proof:}

First, by restricting to the symmetric class of functions, we can adopt the arguments of [22] to show there exists a critical point $\mathbf{P}^{\epsilon}$ of $M_{\epsilon}(\mathbf{P})$. By Lemma $6.1, \mathbf{P}^{\epsilon}=P_{0}+$ $\epsilon \mathbf{a}+o(\epsilon)$ and any other critical point of $M_{\epsilon}(\mathbf{P})$ is in $B_{\delta \epsilon}\left(\mathbf{Q}^{\epsilon}\right)$. 
We now show that $\mathbf{P}^{\epsilon}$ is unique.

By Lemma 6.2, there are only finite number of critical points of $M_{\epsilon}(\mathbf{P})$ in $B_{\delta \epsilon}\left(\mathbf{Q}^{\epsilon}\right)$ (since each critical point is nondegenerate). Let $k_{\epsilon}$ be the number of critical points. At each critical point, we have by Lemma 6.2 ,

$$
\operatorname{deg}\left(\nabla M_{\epsilon}, B_{\delta_{i} \epsilon}\left(\mathbf{Q}_{i}^{\epsilon}\right), 0\right)=(-1)^{5}=-1
$$

where $\delta_{i}>0$ are small constants so that $B_{\delta_{i} \epsilon}\left(\mathbf{Q}_{i}^{\epsilon}\right)$ contains only one critical point (i.e. $\left.\mathbf{Q}_{i}^{\epsilon}\right)$ of $M_{\epsilon}(\mathbf{P})$.

Hence by the additivity of the degree we have

$$
\operatorname{deg}\left(\nabla M_{\epsilon}, B_{\delta \epsilon}\left(\mathbf{Q}^{\epsilon}\right), 0\right)=k_{\epsilon}(-1)^{5} .
$$

On the other hand, it is easy to see that $\tilde{M}_{\epsilon}(\mathbf{P})$ has only one critical point in $B_{\delta \epsilon}\left(\mathbf{Q}^{\epsilon}\right)$ (because of the nondegeneracy of $\left(\nabla^{2} \tilde{M}_{\epsilon}(\mathbf{P})\right)$ ). For $\mathbf{P} \in B_{\delta \epsilon}\left(\mathbf{Q}^{\epsilon}\right)$, we have

$$
\begin{gathered}
e^{-2 d_{P_{i}} / \epsilon}=(1+O(\delta)) e^{-2 d_{Q_{i}}}, w\left(\left|P_{i}-P_{j}\right| / \epsilon\right)=(1+O(\delta)) w\left(\left|Q_{i}^{\epsilon}-Q_{j}^{\epsilon}\right| / \epsilon\right), \\
M_{\epsilon}(\mathbf{P})=(1+O(\delta)) M_{\epsilon}\left(\mathbf{Q}^{\epsilon}\right) .
\end{gathered}
$$

By (1) of Lemma 5.3, we have $\nabla M_{\epsilon}(\mathbf{P})=\nabla \tilde{M}_{\epsilon}(\mathbf{P})+O\left(\tilde{M}_{\epsilon}(\mathbf{P})\right)$. Note that $\nabla M_{\epsilon}(\mathbf{P}) \neq 0$ and $\nabla \tilde{M}_{\epsilon}(\mathbf{P}) \neq 0$ on $\partial B_{\delta \epsilon}\left(\mathbf{Q}^{\epsilon}\right)$. By a continuity argument, we obtain that

$$
\operatorname{deg}\left(\nabla M_{\epsilon}, B_{\delta \epsilon}\left(\mathbf{Q}^{\epsilon}\right), 0\right)=\operatorname{deg}\left(\nabla \tilde{M}_{\epsilon}(\mathbf{P}), B_{\delta \epsilon}\left(\mathbf{Q}^{\epsilon}\right), 0\right)=-1 .
$$

Comparing (6.18) and (6.19), we deduce that $k_{\epsilon}=1$.

Lemma (6.3) shows that type 3.IV solution is unique, up to a rotation, provided that $\epsilon$ is sufficiently small.

\section{Existence And uniqueness of Other types of SOlutions}

In the previous sections, we have proved the uniqueness of type 3.IV solutions. It is easy to see that same techniques (with much simpler computations) show that the type 2.III solutions are unique. It remains to deal with boundary spikes and mixedinterior-boundary spikes. 
To be able to deal with the boundary peak case, we use different approximate functions. (Here the fact that $\Omega=B$ plays an important role.)

We begin with Theorem 1.1 of [30]: for every $P \in \partial \Omega$, there exists a unique boundary spike solution $u_{\epsilon, P}$ which concentrates at $P$. Moreover $u_{\epsilon, P}$ is axially symmetric with respect to the straight line joining 0 and $P$. So for $P \in \partial \Omega$, we may choose our approximate function as follows

$$
w_{\epsilon, P}=u_{\epsilon, P}, \text { for } P \in \partial \Omega .
$$

For $P \in \partial \Omega$, we denote the $i$-th tangential derivative at $P_{j}$ as $\partial_{j, i}=\frac{\partial}{\partial \tau_{P_{j}, i}}, i=$ $1, \ldots, N-1$.

In this case, we see that

$$
S_{\epsilon}\left(w_{\epsilon, P}\right)=0 \text {, if } P \in \partial \Omega \text {. }
$$

Let $\mathbf{P}=\left(P_{1}, \ldots, P_{K}\right)$ be such that $P_{j} \in \partial \Omega, i=1, \ldots, K_{1}, P_{j} \in \Omega, j=K_{1}+1, \ldots, K$. We define a new function (which was introduced first in [23])

$$
\tilde{\varphi}_{K}(\mathbf{P})=\min _{i=1, \ldots, K_{1}, j=1, \ldots, K, i \neq j}\left(\frac{1}{2}\left|P_{i}-P_{j}\right|, \varphi_{K-K_{1}}\left(P_{K_{1}+1}, \ldots, P_{K}\right)\right) .
$$

We can also define $w_{\epsilon, \mathbf{P}}, \mathcal{K}_{\epsilon, \mathbf{P}}, \mathcal{C}_{\epsilon, \mathbf{P}}, \mathcal{K}_{\epsilon, \mathbf{P}}^{\perp}, \mathcal{C}_{\epsilon, \mathbf{P}}^{\perp}, \mathcal{L}_{\epsilon, \mathbf{P}}, v_{\epsilon, \mathbf{P}}, M_{\epsilon}(\mathbf{P})$, etc.

Then similar to Section 4, we will obtain the following

$$
\begin{gathered}
S_{\epsilon}\left(w_{\epsilon, \mathbf{P}}\right)=O\left(e^{-2 \tilde{\varphi}_{K}(\mathbf{P}) / \epsilon}\right) \\
\left\|v_{\epsilon, \mathbf{P}}\right\|_{\epsilon} \leq C e^{-(1+\sigma) \tilde{\varphi}_{K}(\mathbf{P}) / \epsilon} \\
\left\|\partial_{j, i} v_{\epsilon, \mathbf{P}}\right\|_{\epsilon} \leq C \epsilon^{-2} e^{-(1+\sigma) \tilde{\varphi}_{K}(\mathbf{P}) / \epsilon}
\end{gathered}
$$

where $\sigma=\min (1, p-1)$.

Moreover, we have the following equations for the equilibrium positions: for $j=$ $1, \ldots, K_{1}$, we have

$$
\left(\gamma_{1}+o(1)\right) \sum_{l \neq j}\left(\left|P_{l}^{\epsilon}-P_{j}^{\epsilon}\right|\right)^{-\frac{N-1}{2}} e^{-\left|P_{l}^{\epsilon}-P_{j}^{\epsilon}\right| / \epsilon} \partial_{j, i}\left(\left|P_{j}^{\epsilon}-P_{l}^{\epsilon}\right|\right)
$$




$$
+O\left(\epsilon\left(\sum_{j=1}^{K} e^{-2 d_{P_{j}^{\epsilon}} / \epsilon}+\sum_{l \neq k} e^{-\left|P_{l}^{\epsilon}-P_{k}^{\epsilon}\right| / \epsilon}\right)\right)=0, j=1, \ldots, K_{1}, i=1, \ldots, N-1,
$$

(here $\partial_{j, i}$ means the $i-t h$ tangential derivative at $P_{j}^{\epsilon}$ ) and for $j=K_{1}, \ldots, K$ (if $\left|P_{j}^{\epsilon}\right| \geq$ $\left.\delta_{0}\right)$, we have

$$
\begin{gathered}
\left(\gamma_{1}+o(1)\right) c_{N} c\left(P_{j}^{\epsilon}\right) e^{-2 d_{P_{j}^{\epsilon}} / \epsilon}\left(\nabla_{P_{j}^{\epsilon}} d_{P_{j}^{\epsilon}}\right)_{i} \\
+\left(\gamma_{1}+o(1)\right) A_{N} \sum_{l \neq j}\left(\left|P_{l}^{\epsilon}-P_{j}^{\epsilon}\right|\right)^{-\frac{N-1}{2}} e^{-\left|P_{l}^{\epsilon}-P_{j}^{\epsilon}\right| / \epsilon}\left(\nabla_{P_{j}^{\epsilon}}\left|P_{j}^{\epsilon}-P_{l}^{\epsilon}\right|\right)_{i} \\
+O\left(\epsilon\left(\sum_{j=1}^{K} e^{-2 d_{P_{j}^{\epsilon}} / \epsilon}+\sum_{l \neq k} e^{-\left|P_{l}^{\epsilon}-P_{k}^{\epsilon}\right| / \epsilon}\right)\right)=0, j=K_{1}+1, \ldots, K, i=1, \ldots, N .
\end{gathered}
$$

The next lemma is an extension of Lemma 5.4.

Lemma 7.1. Let $K=2$ or 3 and $\tilde{P}_{j}^{\epsilon} \in \Omega, j=1, \ldots, K$ be the $K$ local maximum points of $u_{\epsilon}$. Suppose that $\tilde{P}_{j}^{\epsilon} \rightarrow P_{j}^{0}, j=1, \ldots, K$, then up to a rotation, $\mathbf{P}^{0}=\left(P_{1}^{0}, \ldots, P_{K}^{0}\right)$ must be of of the three types (for $K=2$, Fig. 2) and of the seven types (for $K=3$, Fig. 3).

Proof: As in the proof of Lemma 5.4, by Lemma 5.1, $P_{j}^{0} \neq P_{i}^{0}$ for $i \neq j$.

By Lemma 5.4, we only need to consider boundary spikes or mixed interior and boundary spikes.

Let us consider $K=2$ first. By Theorem 2.1, we may assume that $P_{1}^{\epsilon}, P_{2}^{\epsilon}$ lie on the $x_{1}$-axis. We may further assume that $P_{j}^{\epsilon}=\left(l_{j}^{\epsilon}, 0, \ldots, 0\right), j=1,2, l_{1}^{\epsilon}<l_{2}^{\epsilon}$. If both $P_{1}^{\epsilon}$ and $P_{2}^{\epsilon}$ lie on the boundary, then necessarily by [30], $l_{1}^{\epsilon}=-1, l_{2}^{\epsilon}=1$, which is type 2.I. Suppose that $P_{1}^{\epsilon} \in \partial \Omega$, we may assume that $l_{1}^{\epsilon}=-1$. In this case, (7.8) implies that $\left|P_{1}^{\epsilon}-P_{2}^{\epsilon}\right|=2 d_{P_{2}^{\epsilon}}+o(1)$ and therefore $l_{1}^{\epsilon} \rightarrow \frac{1}{3}$, which is type 2.II.

For $K=3$, by Theorem 2.3, we may assume that $P_{1}^{\epsilon}, P_{2}^{\epsilon}, P_{3}^{\epsilon}$ lie on a two-dimensional plane, say $\left(x_{1}, x_{2}\right)$-plane. That is we have $P_{j}^{\epsilon}=\left(P_{j, 1}^{\epsilon}, P_{j, 2}^{\epsilon}, 0, \ldots, 0\right), j=1,2,3$.

Suppose first we have three boundary spikes: $P_{j}^{\epsilon} \in \partial \Omega, j=1,2,3$. We may assume that $P_{1}^{\epsilon}=(-1,0, \ldots, 0)$. We examine equation $(7.7)$ for $j=1$ and $\partial_{1, i}=\frac{\partial}{\partial P_{1,2}^{\epsilon}}$. Then it 
is easy to see that we must have

$$
e^{-\left|P_{2}^{\epsilon}-P_{1}^{\epsilon}\right| / \epsilon}\left(\frac{P_{2,2}^{\epsilon}-P_{1,2}^{\epsilon}}{\left|P_{2}^{\epsilon}-P_{1}^{\epsilon}\right|}+e^{-\left|P_{3}^{\epsilon}-P_{1}^{\epsilon}\right| / \epsilon}\left(\frac{P_{3,2}^{\epsilon}-P_{1,2}^{\epsilon}}{\left|P_{3}^{\epsilon}-P_{1}^{\epsilon}\right|}+o\left(e^{-\left|P_{2}^{\epsilon}-P_{1}^{\epsilon}\right| / \epsilon}+e^{-\left|P_{3}^{\epsilon}-P_{1}^{\epsilon}\right| / \epsilon}\right)=0\right.\right.
$$

which shows that

$$
\left|P_{2}^{\epsilon}-P_{1}^{\epsilon}\right|=\left|P_{3}^{\epsilon}-P_{1}^{\epsilon}\right|+o(1)
$$

Similarly, we will have

$$
\begin{aligned}
& \left|P_{1}^{\epsilon}-P_{2}^{\epsilon}\right|=\left|P_{3}^{\epsilon}-P_{2}^{\epsilon}\right|+o(1), \\
& \left|P_{1}^{\epsilon}-P_{3}^{\epsilon}\right|=\left|P_{2}^{\epsilon}-P_{3}^{\epsilon}\right|+o(1) .
\end{aligned}
$$

As $\epsilon \rightarrow 0$, it shows that $P_{1}^{0}, P_{2}^{0}$ and $P_{3}^{0}$ must form a perfect triangle, which is type 3.I.

The other cases are similar. We omit the details.

As for the existence, we have

Lemma 7.2. For $\epsilon$ sufficiently small and $K=2$, up to a rotation, there are at least three solutions. The limiting positions are shown in Fig. 2. Moreover, these solutions inherit the partial symmetries of their limiting positions.

Similarly for $\epsilon$ sufficiently small and $K=3$, up to a rotation, there are at least seven solutions. The limiting positions are shown in Fig. 3. Moreover, these solutions inherit the partial symmetries of their limiting positions.

Proof: The existence of all the above solutions follow a general procedure: we first restrict to a symmetric class and then use the idea in [22] and [23]. As an example, we consider the existence of type 2.II solutions. We fix $P_{1}=(-1,0, \ldots, 0)$ and let $P_{2}=\left(l_{2}, 0, \ldots, 0\right),\left|l_{2}-\frac{1}{3}\right|<\delta$. Then we solve the following problem

$$
S_{\epsilon}\left(w_{\epsilon, P_{1}}+w_{\epsilon, P_{2}}+v\right) \in \mathcal{C}_{\epsilon, P_{2}}, \in \mathcal{K}_{\epsilon, P_{2}} \cap H_{\nu, s}^{2}\left(\Omega_{\epsilon}\right),
$$

where

$$
H_{\nu, s}^{2}\left(\Omega_{\epsilon}\right)=\left\{u \in H_{\nu}^{2}\left(\Omega_{\epsilon}\right) \mid u \text { is symmetric with respect to } x_{j}, j=2, \ldots, N\right\} .
$$


Problem (7.10) can be solved since the degeneracy at $P_{1}$ is eliminated by restricting to $H_{\nu, s}^{2}\left(\Omega_{\epsilon}\right)$.

Then we define a reduced energy functional

$$
\tilde{M}_{\epsilon}\left(P_{2}\right)=J_{\epsilon}\left[w_{\epsilon, P_{1}}+w_{\epsilon, P_{2}}+v_{\epsilon}\right]
$$

where $v_{\epsilon}$ is obtained in (7.10). It is easy to compute that asymptotically we have

$$
\tilde{M}_{\epsilon}\left(P_{2}\right)=\epsilon^{N}\left[2 I(w)-\left(c_{1}+o(1)\right) w\left(\left|P_{1}-P_{2}\right| / \epsilon\right)-\left(\frac{\gamma_{1}}{2}+o(1)\right) \varphi_{\epsilon, P_{2}}\left(P_{2}\right)\right]
$$

where $c_{1}$ is some positive constant, $I(w)=\frac{1}{2} \int_{R^{N}}\left(|\nabla w|^{2}+w^{2}\right)-\int_{R^{N}} F(w)$ is the energy of the ground state $w$.

Similar to [22], we now maximize the reduced energy

$$
\max _{\left|l_{2}-\frac{1}{3}\right| \leq \delta} \tilde{M}_{\epsilon}\left(P_{2}\right), P_{2}=\left(l_{2}, 0, \ldots, 0\right) .
$$

Then by the energy expansion of (7.11), it is easy to see that the maximum is attained at some $l_{2}^{\epsilon}$ with $l_{2}^{\epsilon} \rightarrow \frac{1}{3}$ and the corresponding solution $u_{\epsilon}=w_{\epsilon, P_{1}}+w_{\epsilon, P_{2}^{\epsilon}}$ is a solution of type 2.II.

The proof of the existence of the other types of solutions is similar. We omit the details.

As for uniqueness, we consider case by case separately.

The uniqueness of the type 2.I solution is given in [30].

For type 2.II solutions, by Theorem 2.1, we may assume that $P_{1}^{\epsilon}=(-1,0, \ldots, 0), P_{2}^{\epsilon}=$ $\left(l^{\epsilon}, 0, \ldots, 0\right)$. By rotation, we may fix $P_{1}^{\epsilon}$. Moreover, we may consider solutions which are axially symmetric with respect to the $x_{1}$-axis. This reduces the total degrees of freedom into one. Now it is easy to show that $M_{\epsilon}(\mathbf{P})$ has a nondegenerate local maximum at $P_{2}^{\epsilon}$. This shows that the Morse index is 1 and uniqueness follows.

The uniqueness of type 2.III follows by the same proof as the uniqueness of type 3.IV.

Next we consider $K=3$. 
For type 3.I solutions, by Theorem $1.3, P_{j}^{\epsilon}, j=1,2,3$ and the origin must lie in a hyperplane. The problem becomes two-dimensional. Now we fix $P_{1}^{\epsilon}=(-1,0, \ldots, 0)$. Then we have two degrees of freedom. It is easy to see that the Morse index is 2 .

For type 3.II and type 3.III, the proofs are similar.

\section{Appendix A: Proof of Lemma 4.1}

In this appendix, we prove Lemma 4.1 of Section 4.

Recall that $\varphi_{\epsilon, P}(x)$ satisfies

$$
\left\{\begin{array}{l}
\epsilon^{2} \Delta \varphi_{\epsilon, P}(x)-\varphi_{\epsilon, P}(x)=0 \text { in } \Omega, \\
\frac{\partial \varphi_{\epsilon, P}}{\partial \nu}=-\frac{\partial}{\partial \nu} w\left(\frac{x-P}{\epsilon}\right) \text { on } \partial \Omega .
\end{array}\right.
$$

On $\partial \Omega$, we have

$$
\begin{gathered}
\frac{\partial}{\partial \nu} \varphi_{\epsilon, P}(x)=\left(-w^{\prime}\left(\frac{x-P}{\epsilon}\right)\right) \frac{<x-P, \nu>}{|x-P|} \frac{1}{\epsilon} \\
=\epsilon^{-1}(1+O(\epsilon))\left(\frac{\epsilon}{|x-P|}\right)^{\frac{N-1}{2}} e^{-\frac{|x-P|}{\epsilon}} \frac{\langle x-P, \nu>}{|x-P|} .
\end{gathered}
$$

We consider an auxiliary problem (considered in [38] and [50])

$$
\left\{\begin{array}{l}
\epsilon^{2} \Delta \varphi_{\epsilon, P}^{D}(x)-\varphi_{\epsilon, P}^{D}(x)=0 \text { in } \Omega, \\
\varphi_{\epsilon, P}^{D}=w\left(\frac{x-P}{\epsilon}\right) \text { on } \partial \Omega .
\end{array}\right.
$$

Let $\varphi_{\epsilon, P}^{D}=e^{-\Psi_{\epsilon, P}(x) / \epsilon}$, where $\Psi_{\epsilon, P}(x)$ satisfies

$$
\left\{\begin{array}{l}
\epsilon^{2} \Delta \Psi_{\epsilon, P}(x)-\left|\nabla \Psi_{\epsilon, P}(x)\right|^{2}+1=0 \text { in } \Omega, \\
\Psi_{\epsilon, P}=-\epsilon \log w\left(\frac{x-P}{\epsilon}\right) \text { on } \partial \Omega
\end{array}\right.
$$

By Lemma 3.6 of [38], we see that as $\epsilon \rightarrow 0$,

$$
\Psi_{\epsilon, P}(P) \rightarrow 2 d_{P}, \quad \text { as } \epsilon \rightarrow 0 .
$$

It is proved in [50] that

$$
\frac{\partial \Psi_{\epsilon, P}(x)}{\partial \nu}=(-1+O(\epsilon)) \frac{\partial}{\partial \nu}|x-P|=(-1+O(\epsilon)) \frac{<x-P, \nu>}{|x-P|} \text { on } \partial \Omega .
$$


Thus comparing (8.1), (8.2) and (8.6), we obtain that

$$
\varphi_{\epsilon, P}(x)=(1+O(\epsilon)) e^{-\Psi_{\epsilon, P}(x) / \epsilon}, x \in \bar{\Omega} .
$$

Hence $\varphi_{\epsilon, P}(x)=(1+O(\epsilon)) w\left(\frac{x-P}{\epsilon}\right)$ on $\partial \Omega$ and (4.7) of Lemma 4.1 is proved.

(4.8) follows from (8.5).

To compute the exact asymptotic expansion of $\varphi_{\epsilon, P}(P)$, we use the Green's function. Let $G_{\epsilon}(x, z)$ be the Green's function of

$$
\left\{\begin{array}{l}
\epsilon^{2} \Delta G_{\epsilon}(x, z)-G_{\epsilon}(x, z)+\delta(z-x)=0 \text { in } \Omega, \\
\frac{\partial G_{\epsilon}(x, z)}{\partial \nu_{x}}=0 \text { on } \partial \Omega
\end{array}\right.
$$

Then we have

$$
\varphi_{\epsilon, P}(x)=\int_{\partial \Omega} G_{\epsilon}(x, z) \frac{\partial \varphi_{\epsilon, P}}{\partial \nu}(z) d z
$$

We decompose

$$
G_{\epsilon}(x, z)=K_{\epsilon}(|x-z|)+H_{\epsilon}(x, z)
$$

where $K_{\epsilon}(r)$ is the fundamental solution of $\epsilon^{2} \Delta-1$ in $R^{N} \backslash\{0\}$.

Then $H_{\epsilon}$ satisfies

$$
\left\{\begin{array}{l}
\epsilon^{2} \Delta H_{\epsilon}-H_{\epsilon}=0 \text { in } \Omega, \\
\frac{\partial H_{\epsilon}(x, z)}{\partial \nu}=-\frac{\partial K_{\epsilon}(|x-z|)}{\partial \nu} \text { on } \partial \Omega .
\end{array}\right.
$$

Since on $\partial \Omega$, we have

$$
\begin{gathered}
\frac{\partial}{\partial \nu} H_{\epsilon}(x)=\left(-K^{\prime}\left(\frac{x-P}{\epsilon}\right)\right) \frac{<x-P, \nu>1}{|x-P|} \frac{1}{\epsilon} \\
=\epsilon^{-1}\left(d_{N}+O(\epsilon)\right)\left(\frac{\epsilon}{|x-P|}\right)^{\frac{N-1}{2}} e^{-\frac{|x-P|}{\epsilon}} \frac{<x-P, \nu>}{|x-P|}
\end{gathered}
$$

for some generic number $d_{N}>0$.

As before, we have

$$
H_{\epsilon}(x, z)=d_{N} \varphi_{\epsilon, x}(z)=d_{N} w\left(\frac{z-P}{\epsilon}\right) \text { for } z \in \partial \Omega .
$$

So we have

$$
\begin{gathered}
\varphi_{\epsilon, P}(P)=\int_{\partial \Omega}(2+O(\epsilon)) K_{\epsilon}(|z-P|) \frac{\partial \varphi_{\epsilon, P}}{\partial \nu} d z \\
=\left(c_{N}+o(1)\right) \epsilon^{-1} \int_{\partial \Omega}\left(\frac{\epsilon}{|z-P|}\right)^{N-1} e^{-2|z-P| / \epsilon} \frac{<-P, \nu>}{|z-P|} d z
\end{gathered}
$$




$$
=\left(c_{N}+o(1)\right) \epsilon^{N-2} \int_{\partial \Omega}\left(\frac{1}{|z-P|}\right)^{N-1} e^{-2|z-P| / \epsilon} \frac{<z-P, \nu>}{|z-P|} d z .
$$

Let $P$ be such that $|P| \geq d_{0}$ for some $d_{0}>0$. Then the integral in (8.12) is a typical Laplace integral and can computed by the classical Laplace method: namely, we let $z=\sqrt{\epsilon} y$ and then obtain

$$
\varphi_{\epsilon, P}(P)=\left(c_{N}+o(1)\right)\left(d_{P}\left(1-d_{P}\right)\right)^{-\frac{N-1}{2}} \epsilon^{\frac{3 N}{2}-\frac{5}{2}} e^{-2 d_{P} / \epsilon}
$$

for some positive constant $C_{N}>0$. This proves (4.10) of Lemma 4.1.

Next we prove (4.9) of Lemma 4.1. To this end, we note that for $x=P+\epsilon y$

$$
\begin{gathered}
\varphi_{\epsilon, P}(x)=\int_{\partial \Omega} G_{\epsilon}(x, z) \frac{\partial \varphi_{\epsilon, P}}{\partial \nu}(z) d z \\
=\epsilon^{-1}\left(c_{N}+o(1)\right) \int_{\partial \Omega}\left(\frac{\epsilon}{|z-x|}\right)^{-\frac{N-1}{2}}\left(\frac{\epsilon}{|z-P|}\right)^{-\frac{N-1}{2}} e^{-\frac{|z-x|+|z-P|}{\epsilon}} \frac{<z-P, \nu>}{|z-P|} d z \\
=\epsilon^{-1}\left(c_{N}+o(1)\right) \int_{\partial \Omega}\left(\frac{\epsilon}{|z-x|}\right)^{-\frac{N-1}{2}}\left(\frac{\epsilon}{|z-P|}\right)^{-\frac{N-1}{2}} e^{-\frac{2|z-P|}{\epsilon}} e^{-\frac{<z-P, y>}{|z-P|}} \frac{<z-P, \nu>}{|z-P|} d z \\
=(1+o(1)) \varphi_{\epsilon, P}(P) e^{-<\nabla d_{P}, y>}
\end{gathered}
$$

which proves (4.9) of Lemma 4.1.

Finally we prove (4.11) and (4.13) of Lemma 4.1.

For $P \in \Omega$, we define

$$
\Omega_{\epsilon, P}:=\{y \mid \epsilon y+P \in \Omega\} .
$$

If $P=0$, we denote $\Omega_{\epsilon, P}$ as $\Omega_{\epsilon}$.

For $P \in \Omega$, we have

$$
\begin{gathered}
\int_{\Omega} f^{\prime}\left(\bar{w}_{\epsilon, P}\right) \frac{\partial \bar{w}_{\epsilon, P}}{\partial P_{i}} \varphi_{\epsilon, P}(x) d x \\
=(-1+o(1)) \varphi_{\epsilon, P}(P) \epsilon^{N-1} \int_{R^{N}} f^{\prime}(w) \frac{\partial w}{\partial y_{i}} e^{-<\nabla d_{P}, y>} d y \quad \text { (by Lemma 4.1 (2)) } \\
=\left(-\gamma_{1}+o(1)\right) \epsilon^{N-1} \varphi_{\epsilon, P}(P)\left(\nabla d_{P}\right)_{i}+O\left(e^{-(2+\sigma) d_{P} / \epsilon}\right)
\end{gathered}
$$

where $\gamma_{1}$ is given in (4.12). This prove (4.11).

For $P_{1}, P_{2} \in \Omega$ with $\left|P_{1}-P_{2}\right| / \epsilon \rightarrow+\infty$, we have

$$
\int_{\Omega} f^{\prime}\left(\bar{w}_{\epsilon, P_{1}}\right) \bar{w}_{\epsilon, P_{2}} \frac{\partial \bar{w}_{\epsilon, P_{1}}}{\partial P_{1, i}}
$$




$$
\begin{gathered}
=(-1+o(1)) \epsilon^{N-1} \int_{\Omega_{\epsilon, P_{1}}} f^{\prime}(w(y)) \frac{\partial w}{\partial y_{i}} w\left(y+\frac{P_{1}-P_{2}}{\epsilon}\right) d y+O\left(e^{-(1+\sigma)\left|P_{1}-P_{2}\right| / \epsilon}\right) \\
=\epsilon^{N-1} \int_{R^{N}} f(w) \frac{\partial}{\partial y_{i}} w\left(y+\frac{P_{1}-P_{2}}{\epsilon}\right) d y+O\left(e^{-(1+\sigma)\left|P_{1}-P_{2}\right| / \epsilon}\right) \\
(8.15) \quad=\epsilon^{N-1}\left(-\gamma_{1}+o(1)\right) w\left(\frac{\left|P_{1}-P_{2}\right|}{\epsilon}\right)\left(\nabla_{P_{1}}\left(\left|P_{1}-P_{2}\right|\right)\right)_{i}+O\left(e^{-(1+\sigma)\left|P_{1}-P_{2}\right| / \epsilon}\right)
\end{gathered}
$$

which proves (4.13).

\section{Appendix B: Proof of Lemma 4.3}

In this appendix, we prove Lemma 4.3. This is similar to the proof of Lemma 2.4 of $[51]$.

The existence of $v_{\epsilon, \mathbf{P}} \in \mathcal{K}_{\epsilon, \mathbf{P}}^{\perp}$ such that $S_{\epsilon}\left(w_{\epsilon, \mathbf{P}}+v_{\epsilon, \mathbf{P}}\right) \in \mathcal{C}_{\epsilon, \mathbf{P}}$ follows from Section 3 in [22]. The $C^{2}$-smoothness of $v_{\epsilon, \mathbf{P}}$ in $\mathbf{P}$ follows from Lemma 3.5 in [22]. For estimate (4.23), please see Lemma 3.4 of [22]. It remains to estimate $\partial_{j, i} v_{\epsilon, \mathbf{P}}$ and prove (4.24). We decompose

$$
\partial_{j, i} v_{\epsilon, \mathbf{P}}=\sum_{s=1, \ldots, K, t=1, \ldots, N} \alpha_{s t, j i}^{\epsilon} \partial_{s, t} w_{\epsilon, \mathbf{P}}+v_{\epsilon, \mathbf{P}}^{\perp}, v_{\epsilon, \mathbf{P}}^{\perp} \in \mathcal{K}_{\epsilon, \mathbf{P}}^{\perp}
$$

where $\alpha_{s t, j i}^{\epsilon}$ are scalar constants.

We first note that by $(4.23)$

$$
\int_{\Omega_{\epsilon}} \partial_{j, i} v_{\epsilon, \mathbf{P}} \partial_{l, m} w_{\epsilon, \mathbf{P}}=-\int_{\Omega_{\epsilon}} v_{\epsilon, \mathbf{P}} \partial_{j, i} \partial_{l, m} w_{\epsilon, \mathbf{P}}=O\left(\epsilon^{-2} e^{-(1+\sigma) \varphi_{K}(\mathbf{P}) / \epsilon}\right) .
$$

Hence

$$
\sum_{s, t} \alpha_{s t, j i}^{\epsilon} \int_{\Omega_{\epsilon}} \partial_{l, m} w_{\epsilon, \mathbf{P}} \partial_{j, i} w_{\epsilon, \mathbf{P}}=O\left(\epsilon^{-2} e^{-(1+\sigma) \varphi_{K}(\mathbf{P}) / \epsilon}\right)
$$

Since

$$
\int_{\Omega_{\epsilon}} \partial_{l, m} w_{\epsilon, \mathbf{P}} \partial_{s, t} w_{\epsilon, \mathbf{P}}=\epsilon^{-2}\left(\Gamma_{0}+o(1)\right) \delta_{s l} \delta_{m t},
$$

where $\Gamma_{0}=\int_{R^{N}}\left(\frac{\partial w}{\partial y_{1}}\right)^{2}>0$, we obtain $\alpha_{s t, j i}^{\epsilon}=O\left(\epsilon^{-2} e^{-(1+\sigma) \varphi_{K}(\mathbf{P}) / \epsilon}\right)$. 
Next we observe that

$$
S_{\epsilon}\left(w_{\epsilon, \mathbf{P}}+v_{\epsilon, \mathbf{P}}\right)=\sum_{s, t} \beta_{s, t}^{\epsilon}(\mathbf{P}) \partial_{s, t} w_{\epsilon, \mathbf{P}}
$$

where $\beta_{s, t}^{\epsilon}(\mathbf{P}) \in C^{1}$ and $\beta_{s, t}^{\epsilon}(\mathbf{P})=O\left(\epsilon^{-2} e^{-(1+\sigma) \varphi_{K}(\mathbf{P}) / \epsilon}\right)$.

Differentiating the equation (9.1) by $\partial_{l, m}$, we have,

$$
S_{\epsilon}^{\prime}\left(w_{\epsilon, \mathbf{P}}+v_{\epsilon, \mathbf{P}}\right)\left(\partial_{l, m} w_{\epsilon, \mathbf{P}}+\partial_{l, m} v_{\epsilon, \mathbf{P}}\right)-\sum_{s, t} \beta_{s, t}^{\epsilon}(\mathbf{P}) \partial_{l, m} \partial_{s, t} w_{\epsilon, \mathbf{P}} \in \mathcal{C}_{\epsilon, \mathbf{P}}
$$

Substituting the decomposition of $\partial_{j, i} v_{\epsilon, \mathbf{P}}$ into the above equation, we obtain that

$$
\begin{gathered}
S_{\epsilon}^{\prime}\left(w_{\epsilon, \mathbf{P}}+v_{\epsilon, \mathbf{P}}\right)\left(v_{\epsilon, \mathbf{P}}^{\perp}\right)+S_{\epsilon}^{\prime}\left(w_{\epsilon, \mathbf{P}}+v_{\epsilon, \mathbf{P}}\right)\left(\partial_{l, m} w_{\epsilon, \mathbf{P}}+\sum_{s, t} \alpha_{s t, j i}^{\epsilon} \partial_{s, t} w_{\epsilon, \mathbf{P}}\right) \\
-\sum_{s, t} \beta_{s, t}^{\epsilon}(\mathbf{P}) \partial_{s, t} \partial_{l, m} w_{\epsilon, \mathbf{P}} \in \mathcal{C}_{\epsilon, \mathbf{P}} .
\end{gathered}
$$

It is easy to see that

$$
\pi_{\epsilon, \mathbf{P}}^{\perp} \circ S_{\epsilon}^{\prime}\left(w_{\epsilon, \mathbf{P}}+v_{\epsilon, \mathbf{P}}\right): \mathcal{K}_{\epsilon, \mathbf{P}}^{\perp} \rightarrow \mathcal{C}_{\epsilon, \mathbf{P}}^{\perp}
$$

is again invertible for $\epsilon$ sufficiently small. Hence (since $v_{\epsilon, \mathbf{P}}^{\perp} \in \mathcal{K}_{\epsilon, \mathbf{P}}^{\perp}$ )

$$
\begin{gathered}
\left\|v_{\epsilon, \mathbf{P}}^{\perp}\right\|_{H^{2}\left(\Omega_{\epsilon}\right)} \leq C\left\|\pi_{\epsilon, \mathbf{P}}^{\perp} \circ S_{\epsilon}^{\prime}\left(w_{\epsilon, \mathbf{P}}+v_{\epsilon, \mathbf{P}}\right)\left(\partial_{l, m} w_{\epsilon, \mathbf{P}}+\sum_{s, t} \alpha_{s t, j i}^{\epsilon} \partial_{s, t} w_{\epsilon, \mathbf{P}}\right) \mid\right\|_{L^{2}\left(\Omega_{\epsilon}\right)} \\
+C \sum_{s, t}\left|\beta_{s, t}^{\epsilon}(\mathbf{P})\right|\left\|\partial_{s, t} \partial_{l, m} w_{\epsilon, \mathbf{P}}\right\|_{L^{2}\left(\Omega_{\epsilon}\right)} \\
\leq C \epsilon^{-2} e^{-(1+\sigma) \varphi_{K}(\mathbf{P}) / \epsilon}
\end{gathered}
$$

(4.24) is thus proved.

10. Appendix C: Proof of Lemma 5.1

In this appendix, we prove Lemma 5.1. 
We first exclude the case when multiple interior spikes collapse to the boundary. In fact this follows from the proof of Lemma 2.3 of [48]. We prove by contradiction.

Suppose now that $P_{j}^{\epsilon} \in \Omega, \frac{d\left(P_{j}^{\epsilon}, \partial \Omega\right)}{\epsilon} \rightarrow+\infty, P_{j}^{\epsilon} \rightarrow P_{0} \in \partial \Omega, j=1, \ldots, n_{1} \leq K$. Suppose that all the other spikes stay away from $P_{0}$. That is $\left|P_{j}^{\epsilon}-P_{0}\right| \geq \delta_{0}>0$ for $j=n_{2}=1, \ldots, K$.

We recall the following Pohozaev identity: suppose that $u$ satisfies $\epsilon^{2} \Delta u-u+f(u)=0$ in a domain $\Omega$, then for any $y \in R^{N}$, we have

$$
\begin{gathered}
\int_{\Omega_{0}}\left[\left(N F(u)-\frac{N-2}{2} u f(u)\right)-u^{2}\right] \\
=\int_{\partial \Omega_{0}}\left[\left(\epsilon^{2}(x-y, \nabla u) \frac{\partial u}{\partial \nu}-\epsilon^{2}<x-y, \nu>\frac{|\nabla u|^{2}}{2}\right)\right. \\
\left.+<x-y, \nu>\left(\frac{u^{2}}{2}-F(u)\right)+\epsilon^{2} \frac{N-2}{2} u \frac{\partial u}{\partial \nu}\right] .
\end{gathered}
$$

Since $y$ is arbitrary, we deduce that

$$
\int_{\partial \Omega_{0}}\left[\nu\left(\epsilon^{2} \frac{|\nabla u|^{2}}{2}+\frac{u^{2}}{2}-F(u)\right)-\epsilon^{2} \nabla u \frac{\partial u}{\partial \nu}\right]=0 .
$$

Now we choose $\Omega_{0}=\left\{x \in \Omega \mid d\left(x, P_{0}\right) \leq \frac{1}{10}\right\}$ so that $\left|\nu(x)-\nu\left(P_{0}\right)\right| \leq \frac{1}{10}$ where $\nu(x)$ is the normal derivative at $x \in \partial \Omega \cap \partial \Omega_{0}$. Let

$$
\rho_{\epsilon}=\min _{j, k, l=1, \ldots, n_{1}, k \neq l}\left(d\left(P_{j}^{\epsilon}, \partial \Omega\right),\left|P_{k}^{\epsilon}-P_{l}^{\epsilon}\right|\right) / \epsilon .
$$

Then similar to the proof of (2.8) and (2.12) of [48], we have

$$
u_{\epsilon} \geq C e^{-\rho_{\epsilon}} \text { on } \partial \Omega_{0} \cap \partial \Omega
$$

and

$$
u_{\epsilon},\left|\nabla u_{\epsilon}\right| \leq C e^{-\frac{\delta}{\epsilon}} \quad \text { on } \partial \Omega_{0} \backslash \partial \Omega .
$$

Substituting (10.2) and (10.3) into (10.1), we have

$$
\begin{gathered}
0=\int_{\partial \Omega_{0} \cap \partial \Omega}\left[\nu\left(\epsilon^{2} \frac{\left|\nabla u_{\epsilon}\right|^{2}}{2}+\frac{u_{\epsilon}^{2}}{2}-F\left(u_{\epsilon}\right)\right)\right]+O\left(e^{-\delta / \epsilon}\right) \\
\geq C \int_{\partial \Omega_{0} \cap \partial \Omega} u_{\epsilon}^{2}+O\left(e^{-\delta / \epsilon}\right) \geq C e^{-\rho_{\epsilon}}
\end{gathered}
$$

which is a contradiction. 
Second, we exclude the case when multiple interior spikes collapse in the interior. Namely, we have $P_{j}^{\epsilon}, j=1, \ldots, n_{2}, 2 \leq n_{2} \leq K$ where $P_{j}^{\epsilon} \rightarrow P_{0} \in \Omega, j=1, \ldots, n_{2}$. Suppose that all the other spikes stay away from $P_{0}$. That is $\left|P_{j}^{\epsilon}-P_{0}\right| \geq \delta_{0}>0, j=$ $n_{2}+1, \ldots, K$. Similar to the proof of Lemma 3.1 of [46], we see that equation (7.7) remains true: so we have (all the boundary terms are higher order terms)

$$
\sum_{k \neq j} w\left(\left|P_{k}^{\epsilon}-P_{j}^{\epsilon}\right| / \epsilon\right) \nabla_{P_{j}^{\epsilon}}\left(\left|P_{k}^{\epsilon}-P_{j}^{\epsilon}\right|\right)+o\left(\sum_{k \neq j} w\left(\left|P_{k}^{\epsilon}-P_{j}^{\epsilon}\right| / \epsilon\right)\right)=0, j=1, \ldots, n_{2} .
$$

Since $2 \leq n_{2} \leq K \leq 3$, we have either $n_{2}=2$ or $n_{2}=3$.

If $n_{2}=2,(10.5)$ becomes

$$
\begin{gathered}
w\left(\left|P_{1}^{\epsilon}-P_{2}^{\epsilon}\right| / \epsilon\right) \nabla_{P_{1}^{\epsilon}}\left(\left|P_{1}^{\epsilon}-P_{2}^{\epsilon}\right|\right) \\
+o\left(w\left(\left|P_{1}^{\epsilon}-P_{2}^{\epsilon}\right| / \epsilon\right)\right)=0
\end{gathered}
$$

which is impossible.

If $n_{2}=3$, we let $\left|P_{1}^{\epsilon}-P_{2}^{\epsilon}\right|=\min _{i \neq j, i, j=1,2,3}\left|P_{i}^{\epsilon}-P_{j}^{\epsilon}\right|$. We consider two cases. The first case is that $\lim _{\epsilon \rightarrow 0} \frac{w\left(\left|P_{1}^{\epsilon}-P_{2}^{\epsilon}\right| / \epsilon\right)}{w\left(\left|P_{3}^{\epsilon}-P_{2}^{\epsilon}\right| / \epsilon\right)}<+\infty$. In this case, let us assume that $<P_{3}^{\epsilon}-P_{1}^{\epsilon}, P_{2}^{\epsilon}-P_{1}^{\epsilon}>\geq 0$. Then for equation (10.5) at $j=1$, we have

$w\left(\left|P_{1}^{\epsilon}-P_{2}^{\epsilon}\right| / \epsilon\right)+w\left(\left|P_{3}^{\epsilon}-P_{1}^{\epsilon}\right| / \epsilon\right)<\nabla_{P_{1}^{\epsilon}}\left(\left|P_{3}^{\epsilon}-P_{1}^{\epsilon}\right|\right), \nabla_{P_{1}^{\epsilon}}\left(\left|P_{2}^{\epsilon}-P_{1}^{\epsilon}\right|\right)>+o\left(w\left(\left|P_{1}^{\epsilon}-P_{2}^{\epsilon}\right| / \epsilon\right)\right)=0$, which is impossible. If $\lim _{\epsilon \rightarrow 0} \frac{w\left(\left|P_{1}^{\epsilon}-P_{2}^{\epsilon}\right| / \epsilon\right)}{w\left(\left|P_{3}^{\epsilon}-P_{1}^{\epsilon}\right| / \epsilon\right)}<+\infty$, the proof is similar.

The second case is that $\lim _{\epsilon \rightarrow 0} \frac{w\left(\left|P_{3}^{\epsilon}-P_{2}^{\epsilon}\right| / \epsilon\right)}{w\left(\left|P_{1}^{\epsilon}-P_{2}^{\epsilon}\right| / \epsilon\right)}=0$. This reduces to the $n_{2}=2$ case.

Finally, we need to exclude the case when multiple interior spikes collapse to a boundary spike or multiple boundary spikes collapse. That is we have $\left|P_{j}^{\epsilon}-P_{1}^{\epsilon}\right| \rightarrow$ $0, j=2, \ldots, n_{3} \leq K$, where $P_{1}^{\epsilon} \in \partial \Omega$. Without loss of generality, we may assume that $P_{1}^{\epsilon}=P_{0}=(-1, \ldots, 0)$. If $K=2$, by Theorem $2.1, P_{2}^{\epsilon}$ and $P_{1}^{\epsilon}$ are on different sides. Thus, $\left|P_{2}^{\epsilon}-P_{1}^{\epsilon}\right| \geq 1$. So we may assume that $K=3$. By Theorem 2.3, we have $\left|P_{3}^{\epsilon}-P_{1}^{\epsilon}\right| \geq 1$ and $P_{2}^{\epsilon}-P_{1}^{\epsilon} \mid \rightarrow 0$. There are two cases to be considered.

Case 1. $P_{2}^{\epsilon} \in \Omega, P_{2}^{\epsilon} \rightarrow P_{0}=P_{1}^{\epsilon}$.

Let $\tilde{\epsilon}=\frac{\epsilon}{\delta_{\epsilon}}$, where $\delta_{\epsilon}=\left|P_{1}^{\epsilon}-P_{2}^{\epsilon}\right|$. 
Equations (7.7) and (7.8) remains true as long as $\tilde{\varphi}_{K}\left(P_{1}^{\epsilon}, \ldots, P_{K}^{\epsilon}\right) / \epsilon \rightarrow+\infty$. Now we look at equation (7.8) for $j=2, i=1$. We have

$$
\begin{gathered}
2\left(\gamma_{1}+o(1)\right) c\left(P_{2}^{\epsilon}\right) e^{-2 d_{P_{2}^{\epsilon}} / \epsilon}\left(\nabla_{P_{2}^{\epsilon}} d_{P_{2}^{\epsilon}}\right)_{1} \\
\left(\gamma_{1}+o(1)\right)\left(\left|P_{1}^{\epsilon}-P_{2}^{\epsilon}\right|\right)^{-\frac{N-1}{2}} e^{-\left|P_{1}^{\epsilon}-P_{2}^{\epsilon}\right| / \epsilon}\left(\nabla_{P_{2}^{\epsilon}}\left|P_{2}^{\epsilon}-P_{1}^{\epsilon}\right|\right)_{1}=0 .
\end{gathered}
$$

Note that both $\nabla_{P_{2}^{\epsilon}} d_{P_{2}^{\epsilon}}$ and $\nabla_{P_{2}^{\epsilon}}\left(\left|P_{2}^{\epsilon}-P_{1}^{\epsilon}\right|\right)$ are pointing in the same direction. Equation (10.6) can not hold.

Case 2. $P_{1}^{\epsilon} \in \partial \Omega, P_{2}^{\epsilon} \in \partial \Omega,\left|P_{2}^{\epsilon}-P_{1}^{\epsilon}\right| \rightarrow 0$.

In this case, we apply (7.7) to $j=1, \frac{\partial}{\partial \tau_{P_{1}^{\epsilon}}}=\frac{\partial}{\partial x_{1,2}^{\epsilon}}$ and we obtain

$$
\left(\gamma_{1}+o(1)\right)\left(\left|P_{1}^{\epsilon}-P_{2}^{\epsilon}\right|\right)^{-\frac{N-1}{2}} e^{-\left|P_{1}^{\epsilon}-P_{2}^{\epsilon}\right| / \epsilon}\left(\nabla_{P_{2}^{\epsilon}}\left|P_{2}^{\epsilon}-P_{1}^{\epsilon}\right|\right)_{2}=0
$$

which is impossible.

11. Appendix D: Proof of Lemma 5.3

In this appendix, we prove Lemma 5.3.

Proof of (1) and (2) of Lemma 5.3: Observe that

$$
\begin{gathered}
\nabla_{j, i} M_{\epsilon}(\mathbf{P})=<w_{\epsilon, \mathbf{P}}+v_{\epsilon, \mathbf{P}}, \partial_{j, i}\left(w_{\epsilon, \mathbf{P}}+v_{\epsilon, \mathbf{P}}\right)>_{\epsilon}-\epsilon^{-N} \int_{\Omega} f\left(w_{\epsilon, \mathbf{P}}+v_{\epsilon, \mathbf{P}}\right) \partial_{j, i}\left(w_{\epsilon, \mathbf{P}}+v_{\epsilon, \mathbf{P}}\right) \\
=<w_{\epsilon, \mathbf{P}}, \partial_{j, i}\left(w_{\epsilon, \mathbf{P}}\right)>_{\epsilon}-\epsilon^{-N} \int_{\Omega} f\left(w_{\epsilon, \mathbf{P}}\right) \partial_{j, i}\left(w_{\epsilon, \mathbf{P}}\right) \\
+<v_{\epsilon, \mathbf{P}}, \partial_{j, i}\left(w_{\epsilon, \mathbf{P}}\right)>_{\epsilon}-\epsilon^{-N} \int_{\Omega} f^{\prime}\left(w_{\epsilon, \mathbf{P}}\right) v_{\epsilon, \mathbf{P}} \partial_{j, i}\left(w_{\epsilon, \mathbf{P}}\right) \\
+<w_{\epsilon, \mathbf{P}}, \partial_{j, i}\left(v_{\epsilon, \mathbf{P}}\right)>_{\epsilon}-\epsilon^{-N} \int_{\Omega} f\left(w_{\epsilon, \mathbf{P}}\right) \partial_{j, i}\left(v_{\epsilon, \mathbf{P}}\right) \\
+<v_{\epsilon, \mathbf{P}}, \partial_{j, i}\left(v_{\epsilon, \mathbf{P}}\right)>_{\epsilon}-\epsilon^{-N} \int_{\Omega} f\left(w_{\epsilon, \mathbf{P}}\right) \partial_{j, i}\left(v_{\epsilon, \mathbf{P}}\right)+O\left(e^{-(2+\sigma) \varphi_{K}(\mathbf{P}) / \epsilon}\right) \\
=<w_{\epsilon, \mathbf{P}}, \partial_{j, i} w_{\epsilon, \mathbf{P}}>_{\epsilon}-\epsilon^{-N} \int_{\Omega} f\left(w_{\epsilon, \mathbf{P}}\right) \partial_{j, i}\left(w_{\epsilon, \mathbf{P}}\right)+O\left(e^{-(2+\sigma) \varphi_{K}(\mathbf{P}) / \epsilon}\right) \\
=\epsilon^{-N} \int_{\Omega}\left[\sum_{l=1}^{K} f\left(\bar{w}_{\epsilon, P_{l}}\right)-f\left(\sum_{l=1}^{K} w_{\epsilon, P_{l}}\right)\right]\left(\partial_{j, i} w_{\epsilon, \mathbf{P}}\right)+O\left(e^{-(2+\sigma) \varphi_{K}(\mathbf{P}) / \epsilon}\right) \quad \text { (by Lemma 3.1) }
\end{gathered}
$$




$$
\begin{aligned}
& =\epsilon^{-N} \int_{\Omega}\left[\sum_{l=1}^{K}\left(f\left(\bar{w}_{\epsilon, P_{l}}\right)-f\left(w_{\epsilon, P_{l}}\right)\right)-\sum_{l \neq j} f^{\prime}\left(w_{\epsilon, P_{j}}\right) w_{\epsilon, P_{l}}\right] \partial_{j, i} w_{\epsilon, P_{j}}+O\left(e^{-(2+\sigma) \varphi_{K}(\mathbf{P}) / \epsilon}\right) \\
= & \epsilon^{-N} \int_{\Omega}\left[f\left(\bar{w}_{\epsilon, P_{j}}\right)-f\left(w_{\epsilon, P_{j}}\right)\right] \partial_{j, i} w_{\epsilon, P_{j}}-\epsilon^{-N} \sum_{l \neq j} \int_{\Omega} f^{\prime}\left(w_{\epsilon, P_{j}}\right) w_{\epsilon, P_{l}} \partial_{j, i} w_{\epsilon, P_{j}}+O\left(e^{-(2+\sigma) \varphi_{K}(\mathbf{P}) / \epsilon}\right) .
\end{aligned}
$$

$$
=\int_{\Omega_{\epsilon, P_{j}}} f^{\prime}\left(\bar{w}_{\epsilon, P_{j}}\right)\left(-\varphi_{\epsilon, P_{j}}\right) \partial_{j, i} \bar{w}_{\epsilon, P_{j}}-\sum_{l \neq j} \int_{\Omega_{\epsilon, P_{j}}} f^{\prime}\left(\bar{w}_{\epsilon, P_{j}}\right) \bar{w}_{\epsilon, P_{l}} \partial_{j, i} \bar{w}_{\epsilon, P_{j}}+O\left(e^{-(2+\sigma) \varphi_{K}(\mathbf{P}) / \epsilon}\right)
$$

We discuss two cases.

Case 1. If $\left|P_{j}^{\epsilon}\right| \leq \frac{1}{10}$, then the first term in (11.1)

$$
\int_{\Omega_{\epsilon, P_{j}}} f^{\prime}\left(\bar{w}_{\epsilon, P_{j}}\right)\left(-\varphi_{\epsilon, P_{j}}\right) \partial_{j, i} \bar{w}_{\epsilon, P_{j}}=O\left(e^{-2 d_{P_{j}^{\epsilon}}} / \epsilon\right)
$$

which is a higher order term, comparing with the second order term (since $\left|P_{k}^{\epsilon}-P_{j}^{\epsilon}\right| \leq$ $\left.\frac{11}{10}<2 d_{P_{j}^{\epsilon}}\right)$. This proves $(5.8)$ of Lemma 5.3.

Case 2. If $\left|P_{j}^{\epsilon}\right| \geq d_{0}$ for some $d_{0}>0$, then (11.1) equals $(11.2) \epsilon^{N-1}\left(\gamma_{1}+o(1)\right) \varphi_{\epsilon, P_{j}}\left(P_{j}\right)\left(\nabla d_{P_{j}}\right)_{i}+\epsilon^{N-1}\left(\gamma_{1}+o(1)\right) \sum_{l \neq j} w\left(\left|P_{j}-P_{l}\right| / \epsilon\right)\left(\nabla\left|P_{j}-P_{l}\right|\right)_{i}$ by (4.11) and (4.13) of Lemma 4.1.

By using Lemma 4.1, we see that (5.7) holds.

Proof of (3) of Lemma 5.3: Let $\mathbf{P}^{\epsilon}$ be a critical point of $M_{\epsilon}(\mathbf{P})$ in $\Lambda_{\delta}$ such that $\left|P_{j}^{\epsilon}\right| \geq d_{0}, j=1, \ldots, K$ for some $d_{0}>0$. We now expand,

$$
\begin{aligned}
& \left.\partial_{l, m} \partial_{j, i} M_{\epsilon}(\mathbf{P})\right|_{\mathbf{P}=\mathbf{P}^{\epsilon}} \\
& =<\partial_{l, m}\left(w_{\epsilon, \mathbf{P}}+v_{\epsilon, \mathbf{P}}\right), \partial_{j, i}\left(w_{\epsilon, \mathbf{P}}+v_{\epsilon, \mathbf{P}}\right)>\left._{\epsilon}\right|_{\mathbf{P}=\mathbf{P}^{\epsilon}} \\
& +<w_{\epsilon, \mathbf{P}}+v_{\epsilon, \mathbf{P}}, \partial_{l, m} \partial_{j, i}\left(w_{\epsilon, \mathbf{P}}+v_{\epsilon, \mathbf{P}}\right)>_{\epsilon} \mid \mathbf{P}=\mathbf{P}^{\epsilon} \\
& -\left.\left.\epsilon^{-N} \int_{\Omega} f^{\prime}\left(w_{\epsilon, \mathbf{P}^{\epsilon}}+v_{\epsilon, \mathbf{P}^{\epsilon}}\right) \partial_{l, m}\left(w_{\epsilon, \mathbf{P}}+v_{\epsilon, \mathbf{P}}\right)\right|_{\mathbf{P}=\mathbf{P}^{\epsilon}} \partial_{j, i}\left(w_{\epsilon, \mathbf{P}}+v_{\epsilon, \mathbf{P}}\right)\right|_{\mathbf{P}=\mathbf{P}^{\epsilon}} \\
& -\left.\epsilon^{-N} \int_{\Omega} f\left(w_{\epsilon, \mathbf{P}^{\epsilon}}+v_{\epsilon, \mathbf{P}^{\epsilon}}\right) \partial_{l, m} \partial_{j, i}\left(w_{\epsilon, \mathbf{P}}+v_{\epsilon, \mathbf{P}}\right)\right|_{\mathbf{P}=\mathbf{P}^{\epsilon}} \\
& =<\partial_{l, m}\left(w_{\epsilon, \mathbf{P}}+v_{\epsilon, \mathbf{P}}\right), \partial_{j, i}\left(w_{\epsilon, \mathbf{P}}+v_{\epsilon, \mathbf{P}}\right)>_{\epsilon} \mid \mathbf{P}=\mathbf{P}^{\epsilon}
\end{aligned}
$$




$$
-\left.\left.\epsilon^{-N} \int_{\Omega} f^{\prime}\left(w_{\epsilon, \mathbf{P}^{\epsilon}}+v_{\epsilon, \mathbf{P}^{\epsilon}}\right) \partial_{l, m}\left(w_{\epsilon, \mathbf{P}}+v_{\epsilon, \mathbf{P}}\right)\right|_{\mathbf{P}=\mathbf{P}^{\epsilon}} \partial_{j, i}\left(w_{\epsilon, \mathbf{P}}+v_{\epsilon, \mathbf{P}}\right)\right|_{\mathbf{P}=\mathbf{P}^{\epsilon}}
$$

(since $\mathbf{P}^{\epsilon}$ is a critical point of $M_{\epsilon}(\mathbf{P})$ )

$$
\begin{aligned}
& =<\partial_{l, m} w_{\epsilon, \mathbf{P}}, \partial_{j, i} w_{\epsilon, \mathbf{P}}>\left._{\epsilon}\left|\mathbf{P}=\mathbf{P}^{\epsilon}-\epsilon^{-N} \int_{\Omega} f^{\prime}\left(w_{\epsilon, \mathbf{P}^{\epsilon}}+v_{\epsilon, \mathbf{P}^{\epsilon}}\right) \partial_{l, m} w_{\epsilon, \mathbf{P}}\right|_{\mathbf{P}=\mathbf{P} \epsilon} \partial_{j, i} w_{\epsilon, \mathbf{P}}\right|_{\mathbf{P}=\mathbf{P}^{\epsilon}} \\
& +<\partial_{l, m} w_{\epsilon, \mathbf{P}}, \partial_{j, i} v_{\epsilon, \mathbf{P}}>\left._{\epsilon}\left|\mathbf{P}=\mathbf{P}^{\epsilon}-\epsilon^{-N} \int_{\Omega} f^{\prime}\left(w_{\epsilon, \mathbf{P}^{\epsilon}}+v_{\epsilon, \mathbf{P}^{\epsilon}}\right) \partial_{l, m} w_{\epsilon, \mathbf{P}}\right|_{\mathbf{P}=\mathbf{P}^{\epsilon}} \partial_{j, i} v_{\epsilon, \mathbf{P}}\right|_{\mathbf{P}=\mathbf{P}^{\epsilon}} \\
& +<\partial_{l, m} v_{\epsilon, \mathbf{P}}, \partial_{j, i} w_{\epsilon, \mathbf{P}}>\left._{\epsilon}\left|\mathbf{P}=\mathbf{P}^{\epsilon}-\epsilon^{-N} \int_{\Omega} f^{\prime}\left(w_{\epsilon, \mathbf{P}^{\epsilon}}+v_{\epsilon, \mathbf{P}^{\epsilon}}\right) \partial_{l, m} v_{\epsilon, \mathbf{P}}\right|_{\mathbf{P}=\mathbf{P}^{\epsilon}} \partial_{j, i} w_{\epsilon, \mathbf{P}}\right|_{\mathbf{P}=\mathbf{P}^{\epsilon}} \\
& +<\partial_{l, m} v_{\epsilon, \mathbf{P}}, \partial_{j, i} v_{\epsilon, \mathbf{P}}>\left._{\epsilon}\left|\mathbf{P}=\mathbf{P}^{\epsilon}-\epsilon^{-N} \int_{\Omega} f^{\prime}\left(w_{\epsilon, \mathbf{P}^{\epsilon}}+v_{\epsilon, \mathbf{P}^{\epsilon}}\right) \partial_{l, m} v_{\epsilon, \mathbf{P}}\right|_{\mathbf{P}=\mathbf{P}^{\epsilon}} \partial_{j, i} v_{\epsilon, \mathbf{P}}\right|_{\mathbf{P}=\mathbf{P}^{\epsilon}} \\
& =I_{1}+I_{2}+I_{3}+I_{4}
\end{aligned}
$$

where $I_{i}, i=1, \ldots, 4$ are defined at the last equality.

We now estimate each term. Certainly the estimate of $I_{2}$ is the same as that of $I_{3}$.

By Lemma 4.3,

$$
I_{4}=O\left(\left\|\partial_{l, m} v_{\epsilon, \mathbf{P}^{\epsilon}}\right\|_{\epsilon}\left\|\partial_{j, i} v_{\epsilon, \mathbf{P}^{\epsilon}}\right\|_{\epsilon}\right)=O\left(e^{-(2+\sigma) \varphi_{K}\left(\mathbf{P}^{\epsilon}\right) / \epsilon}\right) .
$$

Next we consider $I_{2}$ :

$$
I_{2}=\epsilon^{-N} \int_{\Omega}\left[f\left(\bar{w}_{\epsilon, P_{l}^{\epsilon}}\right) \partial_{l, m} \bar{w}_{\epsilon, P_{l}^{\epsilon}}-f\left(w_{\epsilon, \mathbf{P}^{\epsilon}}+v_{\epsilon, \mathbf{P}^{\epsilon}}\right) \partial_{l, m} w_{\epsilon, P_{l}^{\epsilon}}\right] \partial_{j, i} w_{\epsilon, P_{j}^{\epsilon}}=O\left(e^{-(2+\sigma) \varphi_{K}\left(\mathbf{P}^{\epsilon}\right) / \epsilon}\right) .
$$

Similarly, we have

$$
I_{3}=O\left(e^{-(2+\sigma) \varphi_{K}\left(\mathbf{P}^{\epsilon}\right) / \epsilon}\right) .
$$

Hence it remains to compute $I_{1}$ only. We divide it into two cases: $j \neq l$ and $j=l$.

When $j \neq l$, we have by Lemma 4.1

$$
\begin{gathered}
I_{1}=\epsilon^{-N} \int_{\Omega}\left[f^{\prime}\left(\bar{w}_{\epsilon, P_{l}^{\epsilon}}\right) \partial_{l, m} \bar{w}_{\epsilon, P_{l}^{\epsilon}}-f^{\prime}\left(w_{\epsilon, \mathbf{P}^{\epsilon}}\right) \partial_{l, m} w_{\epsilon, P_{l}^{\epsilon}}\right] \partial_{j, i} w_{\epsilon, P_{j}^{\epsilon}} \\
=\epsilon^{-N} \int_{\Omega}\left[f^{\prime}\left(\bar{w}_{\epsilon, P_{l}^{\epsilon}}\right) \partial_{l, m} \bar{w}_{\epsilon, P_{l}^{\epsilon}}-\left(f^{\prime}\left(w_{\epsilon, P_{l}^{\epsilon}}\right)+f^{\prime}\left(w_{\epsilon, P_{j}^{\epsilon}}\right)\right) \partial_{l, m} w_{\epsilon, P_{l}^{\epsilon}}\right] \partial_{j, i} \bar{w}_{\epsilon, P_{j}^{\epsilon}}+O\left(e^{-(2+\sigma) \varphi_{K}\left(\mathbf{P}^{\epsilon}\right) / \epsilon}\right) \\
=\epsilon^{-N} \int_{\Omega}\left[f^{\prime}\left(\bar{w}_{\epsilon, P_{l}^{\epsilon}}\right) \partial_{l, m} \bar{w}_{\epsilon, P_{l}^{\epsilon}}-f^{\prime}\left(w_{\epsilon, P_{l}^{\epsilon}}\right) \partial_{l, m} w_{\epsilon, P_{l}^{\epsilon}}\right] \partial_{j, i} \bar{w}_{\epsilon, P_{j}^{\epsilon}} \\
-\epsilon^{-N} \int_{\Omega} f^{\prime}\left(w_{\epsilon, P_{j}^{\epsilon}}\right) \partial_{l, m} w_{\epsilon, P_{l}^{\epsilon}} \partial_{j, i} \bar{w}_{\epsilon, P_{j}^{\epsilon}}+O\left(e^{-(2+\sigma) \varphi_{K}\left(\mathbf{P}^{\epsilon}\right) / \epsilon}\right) \\
=-\epsilon^{-N} \int_{\Omega} f^{\prime}\left(w_{\epsilon, P_{j}^{\epsilon}}\right) \partial_{l, m} w_{\epsilon, P_{l}^{\epsilon}} \partial_{j, i} \bar{w}_{\epsilon, P_{j}^{\epsilon}}+O\left(e^{-(2+\sigma) \varphi_{K}\left(\mathbf{P}^{\epsilon}\right) / \epsilon}\right)
\end{gathered}
$$




$$
\begin{array}{r}
=\epsilon^{-2} w\left(\frac{\left|P_{j}^{\epsilon}-P_{l}^{\epsilon}\right|}{\epsilon}\right) \int_{\Omega_{\epsilon, P_{j}^{\epsilon}}} f^{\prime}(w) \frac{\partial w}{\partial y_{i}} e^{-<e_{j l}^{\epsilon}, y>}\left(e_{j l}^{\epsilon}\right)_{m}+O\left(e^{-(2+\sigma) \varphi_{K}\left(\mathbf{P}^{\epsilon}\right) / \epsilon}\right) \\
=\epsilon^{-2} w\left(\left|P_{j}^{\epsilon}-P_{l}^{\epsilon}\right| / \epsilon\right)\left(\gamma_{1}+o(1)\right)\left(e_{j l}^{\epsilon}\right)_{m}\left(e_{j l}^{\epsilon}\right)_{i}+O\left(e^{-(2+\sigma) \varphi_{K}\left(\mathbf{P}^{\epsilon}\right) / \epsilon}\right)
\end{array}
$$

For $j=l$, we have

$$
\begin{gathered}
I_{1}=\epsilon^{-N} \int_{\Omega}\left[f^{\prime}\left(\bar{w}_{\epsilon, P_{j}^{\epsilon}}\right) \partial_{j, m} \bar{w}_{\epsilon, P_{j}^{\epsilon}}-f^{\prime}\left(w_{\epsilon, \mathbf{P}^{\epsilon}}\right) \partial_{j, m} w_{\epsilon, P_{j}^{\epsilon}}\right] \partial_{j, i} w_{\epsilon, P_{j}^{\epsilon}} \\
=\epsilon^{-N} \int_{\Omega}\left[f^{\prime}\left(\bar{w}_{\epsilon, P_{j}^{\epsilon}}\right) \partial_{j, m} \bar{w}_{\epsilon, P_{j}^{\epsilon}}-f^{\prime}\left(w_{\epsilon, P_{j}^{\epsilon}}\right) \partial_{j, m} w_{\epsilon, P_{j}^{\epsilon}}\right] \partial_{j, i} w_{\epsilon, P_{j}^{\epsilon}} \\
-\epsilon^{-N} \sum_{k \neq j} \int_{\Omega} f^{\prime \prime}\left(\bar{w}_{\epsilon, P_{j}^{\epsilon}}\right) w_{\epsilon, P_{k}^{\epsilon}} \partial_{j, m} w_{\epsilon, P_{j}^{\epsilon}} \partial_{j, i} w_{\epsilon, P_{j}^{\epsilon}}+O\left(e^{-(2+\sigma) \varphi_{K}\left(\mathbf{P}^{\epsilon}\right) / \epsilon}\right) \\
=I_{1,1}-I_{1,2} .
\end{gathered}
$$

For $I_{1,1}$, we have

$$
\begin{gathered}
I_{1,1}=\epsilon^{-N} \int_{\Omega}\left[\partial_{j, m} f\left(\bar{w}_{\epsilon, P_{j}^{\epsilon}}\right)-\partial_{j, m} f\left(w_{\epsilon, P_{j}^{\epsilon}}\right)\right] \partial_{j, i} \bar{w}_{\epsilon, P_{j}^{\epsilon}} \\
=\epsilon^{-N} \int_{\Omega}\left[\left(-\frac{\partial}{\partial x_{m}} f\left(\bar{w}_{\epsilon, P_{j}^{\epsilon}}\right)-f\left(w_{\epsilon, P_{j}^{\epsilon}}\right)\right)\right]\left(-\frac{\partial}{\partial x_{i}} \bar{w}_{\epsilon, P_{j}^{\epsilon}}+O\left(e^{-(2+\sigma) \varphi_{K}\left(\mathbf{P}^{\epsilon}\right) / \epsilon}\right)\right. \\
=\epsilon^{-N} \int_{\Omega}\left(f\left(w_{\epsilon, P_{j}^{\epsilon}}\right)-f\left(\bar{w}_{\epsilon, P_{j}^{\epsilon}}\right)\left(\frac{\partial^{2}}{\partial x_{i} \partial x_{m}} \bar{w}_{\epsilon, P_{j}^{\epsilon}}\right)++O\left(e^{-(2+\sigma) \varphi_{K}\left(\mathbf{P}^{\epsilon}\right) / \epsilon}\right)\right. \\
=\epsilon^{-2} \int_{\Omega_{\epsilon, P_{j}^{\epsilon}}} f^{\prime}(w)(y) \varphi_{\epsilon, P_{j}^{\epsilon}}\left(P_{j}^{\epsilon}+\epsilon y\right) \frac{\partial^{2} w}{\partial y_{i} \partial y_{m}}++O\left(e^{-(2+\sigma) \varphi_{K}\left(\mathbf{P}^{\epsilon}\right) / \epsilon}\right) \\
=\epsilon^{-2} \varphi_{\epsilon, P_{j}^{\epsilon}}\left(P_{j}^{\epsilon}\right) \int_{R^{N}} f^{\prime}(w) e^{-<\nabla_{P_{j}^{\epsilon}} d_{P_{j}^{\epsilon}, y>}} \frac{\partial^{2} w}{\partial y_{i} \partial y_{m}} d y++O\left(e^{-(2+\sigma) \varphi_{K}\left(\mathbf{P}^{\epsilon}\right) / \epsilon}\right)
\end{gathered}
$$

(by (4.9) of Lemma 4.1)

$$
\begin{aligned}
&=\epsilon^{-2} \varphi_{\epsilon, P_{j}^{\epsilon}}\left(P_{j}^{\epsilon}\right) \int_{R^{N}}(-f^{\prime \prime}(w) \frac{\partial w}{\partial y_{i}} \frac{\partial w}{\partial y_{m}} e^{-<\nabla_{P_{j}^{\epsilon}} d_{P_{j}^{\epsilon}}, y>} d y++O\left(e^{-(2+\sigma) \varphi_{K}\left(\mathbf{P}^{\epsilon}\right) / \epsilon}\right) \\
&=\epsilon^{-2} \varphi_{\epsilon, P_{j}^{\epsilon}}\left(P_{j}^{\epsilon}\right) e_{j, i}^{\epsilon} e_{j, m}^{\epsilon}\left(-\gamma_{1}+o(1)\right)
\end{aligned}
$$

For $I_{1,2}$, we have

$$
\begin{gathered}
I_{1,2}=\epsilon^{-N} \sum_{k j} \int_{\Omega} f^{\prime \prime}\left(\bar{w}_{\epsilon, P_{j}^{\epsilon}}\right) \bar{w}_{\epsilon, P_{k}^{\epsilon}} \partial_{j, m} \bar{w}_{\epsilon, P_{j}^{\epsilon}} \partial_{j, i} \bar{w}_{\epsilon, P_{j}^{\epsilon}}+O\left(e^{-(2+\sigma) \varphi_{K}\left(\mathbf{P}^{\epsilon}\right) / \epsilon}\right) \\
=\epsilon^{-2} \sum_{k \neq j} \int_{R^{N}} f^{\prime \prime}(w) \frac{\partial w}{\partial y_{i}} \frac{\partial w}{\partial y_{m}} w\left(y+\frac{P_{j}^{\epsilon}-P_{k}^{\epsilon}}{\epsilon}\right) d y+O\left(e^{-(2+\sigma) \varphi_{K}\left(\mathbf{P}^{\epsilon}\right) / \epsilon}\right)
\end{gathered}
$$




$$
=\epsilon^{-2} \sum_{k \neq j} w\left(\frac{\left|P_{j}^{\epsilon}-P_{k}^{\epsilon}\right|}{\epsilon}\right) e_{j k, i}^{\epsilon} e_{j k, m}^{\epsilon}\left(\gamma_{1}+o(1)\right) .
$$

Combining all together, we have

$$
\begin{gathered}
\left.\partial_{l, m} \partial_{j, i} M_{\epsilon}(\mathbf{P})\right|_{\mathbf{P}=\mathbf{P}^{\epsilon}} \\
=\epsilon^{-2}\left(\gamma_{1}+o(1)\right) w\left(\frac{\left|P_{j}^{\epsilon}-P_{k}^{\epsilon}\right|}{\epsilon}\right) e_{j l, m}^{\epsilon} e_{j l, i}^{\epsilon}\left(1-\delta_{j l}\right) \\
-\epsilon^{-2}\left(\gamma_{1}+o(1)\right) \varphi_{\epsilon, P_{j}^{\epsilon}}\left(P_{j}^{\epsilon}\right) e_{j, i}^{\epsilon} e_{l, m}^{\epsilon} \delta_{j l} \\
-\epsilon^{-2}\left(\gamma_{1}+o(1)\right) \sum_{k \neq j} w\left(\frac{\left|P_{j}^{\epsilon}-P_{k}^{\epsilon}\right|}{\epsilon}\right) e_{j k, i}^{\epsilon} e_{j k, m}^{\epsilon} \delta_{j l},
\end{gathered}
$$

which is exactly (5.9).

\section{ACKNOWLEDGMENTS}

This research of the second author is partially supported by an Earmarked Grant from RGC of Hong Kong. This work was done while the second author visited the National Center for Theoretical Sciences, Taiwan. He would like to thank the center for its kind hospitality.

\section{REFERENCES}

[1] N. Alikakos, G. Fusco and M. Kowalczyk, Finite dimensional dynamics and interfaces intersecting the boundary I, Indiana Univ. Math. J. 45(1996), 1119-1155.

[2] N. Alikakos and G. Fusco, Slow dynamics for the Cahn-Hilliard equation in higher space dimensions: the motion of bubbles, Arch. Rational Mech. Anal. 141 (1998), no. 1, 1-61.

[3] N. Alikakos, L. Bronsard and G. Fusco, Slow motion in the gradient theory of phase transitions via energy and spectrum, Calc. Var. Partial Differential Equations 6 (1998), no. 1, 39-66.

[4] N. Alikakos and M. Kowalczyk, Critical points of a singular perturbation problem via reduced energy and local linking, J. Diff. Eqns. 159(1999), 403-426.

[5] P. Bates, E.N. Dancer and J. Shi, Multi-spike stationary solutions of the Cahn-Hilliard equation in higher-dimension and instability, Adv. Differential Equations 4 (1999), 1-69.

[6] P. Bates and G. Fusco, Equilibria with many nuclei for the Cahn-Hilliard equation, J. Diff. Eqns. 4(1999), 1-69.

[7] P. Bates and J. Shi, Existence and instability of spike layer solutions to singular perturbation problems, J. Funct. Anal., to appear.

[8] A. Bahri and J. M. Coron, On a nonlinear elliptic equation involving the critical Sobolev exponent: the effect of the topology of the domain, Comm. Pure Appl. Math. 41(1988), 255-294.

[9] C.C. Chen and C.S. Lin, Uniqueness of the ground state solution of $\Delta u+f(u)=0$ in $R^{N}, N \geq 3$, Comm. PDE. 16(1991), 1549-1572. 
[10] C.C. Chen and C.S. Lin, On the symmetry of blowup solutions to a mean field equation, Ann. Inst. H. Poincare Anal. Non Lineaire 18 (2001), 271-296.

[11] G. Cerami and J. Wei, Multiplicity of multiple interior spike solutions for some singularly perturbed Neumann problem, International Math. Research Notes 12 (1998), 601-626.

[12] E.N. Dancer and J. Wei, On the effect of domain topology in some singular perturbation problems, Topological Methods in Nonlinear Analysis 11(2) (1998), 227-248.

[13] E.N. Dancer and S. Yan, Multipeak solutions for a singular perturbed Neumann problem, Pacific J. Math. 189(1999), 241-262.

[14] E.N. Dancer and S. Yan, Interior and boundary peak solutions for a mixed boundary value problem, Indiana Univ. Math. J. 48 (1999), 1177-1212.

[15] M. del Pino, P. Felmer and J. Wei, On the role of mean curvature in some singularly perturbed Neumann problems, SIAM J. Math. Anal. 31 (1999), 63-79.

[16] M. del Pino, P. Felmer and J. Wei, On the role of distance function in some singularly perturbed problems, Comm. PDE 25(2000), 155-177.

[17] Floer, A. and Weinstein, A., Nonspreading wave packets for the cubic Schrödinger equation with a bounded potential, J. Funct. Anal. 69 (1986), 397-408.

[18] A. Gierer and H. Meinhardt, A theory of biological pattern formation. Kybernetik (Berlin) 12(1972), 30-39.

[19] B. Gidas, W.M. Ni, and L. Nirenberg, Symmetry of positive solutions of nonlinear elliptic equations in $R^{N}$, Adv. Math. Suppl Stud. 7A (1981), 369-402.

[20] M. Grossi, Uniqueness of least-energy solution for a semilinear Neumann problem, Proc. Amer. Math. Soc. 128(2000), 1665-1672.

[21] M. Grossi, A. Pistoia and J. Wei, Existence of multipeak solutions for a semilinear Neumann problem via nonsmooth critical point theory, Cal. Var. PDE 11(2000) 143-175.

[22] C. Gui and J. Wei, Multiple interior peak solutions for some singular perturbation problems, $J$. Diff. Eqns. 158(1999), 1-27.

[23] C. Gui and J. Wei, On multiple mixed interior and boundary peak solutions for some singularly perturbed Neumann problems, Can. J. Math. 52(2000), 522-538.

[24] C. Gui, J. Wei and M. Winter, Multiple boundary peak solutions for some singularly perturbed Neumann problems, Analyse Non Linearie, Annoles de l'Institut H. Poincare 17(2000), 47-82.

[25] M. Kowalczyk, Multiple spike layers in the shadow Gierer-Meinhardt system: existence of equilibria and approximate invariant manifold, Duke Math. J. 98(1999), 59-111.

[26] M.K. Kwong, Uniquness of positive solutions of $\Delta u-u+u^{p}=0$ in $R^{N}$, Arch. Rational Mech. Anal. 105(1991), 243-266.

[27] Y.-Y. Li, On a singularly perturbed equation with Neumann boundary condition, Comm. PDE 23(1998), 487-545.

[28] Y.-Y. Li and L. Nirenberg, The Dirichlet problem for singularly perturbed elliptic equations, Comm. Pure Appl. Math. 51 (1998), 1445-1490.

[29] C.-S. Lin, Locating the peaks of solutions via the maximum principle, I. Neumann probelm, Comm. Pure Appl. Math. 54 (2001), no. 9, 1065-1095.

[30] C.-S. Lin and I. Takagi, Method of rotating planes applied to a singularly perturbed Neumann problem, Cal. Var. PDE 2001, to appear.

[31] C.-S. Lin and W.-M. Ni, On the diffusion coefficient of a semilinear Neumann problem, Calculus of variations and partial differential equations (Trento, 1986) 160-174, Lecture Notes in Math., 1340, Springer, Berlin-New York, 1988.

[32] C.-S. Lin, W.-M. Ni and I. Takagi, Large amplitude stationary solutions to a chemotaxis systems, J. Diff. Eqns 72 (1988), 1-27.

[33] W.-M. Ni, Diffusion, cross-diffusion, and their spike-layer steady states, Notices of Amer. Math. soc. 45(1998), 9-18. 
[34] W.-M. Ni and I. Takagi, On the shape of least energy solution to a semilinear Neumann problem, Comm. Pure Appl. Math. 41(1991), 819-851.

[35] W.-M. Ni and I. Takagi, Locating the peaks of least energy solutions to a semilinear Neumann problem, Duke Math. J. 70 (1993), 247-281.

[36] W.-M. Ni and I. Takagi, Point-condensation generated by a reaction-diffusion system in axially symmetric domains, Japan J. Industrial Appl. Math. 12(1995), 327-365.

[37] W.-M. Ni, I. Takagi and J. Wei, On the location and profile of spike-layer solutions to singularly perturbed semilinear Dirichlet problems: intermediate solutions, Duke Math. J. 94 (1998), 597618.

[38] W.-M. Ni and J. Wei, On the location and profile of spike-layer solutions to singularly perturbed semilinear Dirichlet problems, Comm. Pure Appl. Math. 48 (1995), 731-768.

[39] W.-M. Ni, Diffusion, cross-diffusion, and their spike-layer steady states, Notices of Amer. Math. Soc. 45 (1998), 9-18.

[40] Y.G. Oh, Existence of semi-classical bound states of nonlinear Schrödinger equations with potentials of the class $(V)_{a}$, Comm. PDE 13 (12) (1988), 1499-1519.

[41] Y.G. Oh, On positive multi-bump bound states of nonlinear Schrödinger equations under multiple-well potentials, Comm. Math. Phys. 131 (1990), 223-253.

[42] O. Rey, The role of Green's function in a nonlinear elliptic equation involving the critical Sobolev exponent, J. Funct. Anal. 89(1990), 1-52.

[43] L.A. Peletier and J. Serrin, Uniqueness of positive solutions of semilinear equations in $R^{N}$, Arch. Rational Mech. Anal. 81(1983), 181-197.

[44] J. Wei, On the construction of single-peaked solutions to a singularly perturbed semilinear Dirichlet problem, J. Differential Equations 129 (1996), 315-333.

[45] J. Wei, On the boundary apikw layer solutions of singularly perturbed semilinear Neumann problem, J. Diffential Equations 134 (1997), 104-133.

[46] J. Wei, Conditions for two-peaked solutions of singularly perturbed elliptic equations, Manuscripta Mathematica, 96(1998), 113-136.

[47] J. Wei, On the interior spike layer solutions for some singular perturbation problems, Proc. Royal Soc. Edinburgh, Section A (Mathematics) 128(1998), 849-874.

[48] J. Wei, On the interior spike layer solutions of singularly perturbed semilinear Neumann problem, Tohoku Math. J. 50(1998), 159-178.

[49] J. Wei, Uniqueness and eigenvalue estimates of boundary spike solutions, Proc. R. Soc. Edinburgh Sect. A 131(2001), 1457-1480.

[50] J. Wei, On the effect of domain geometry in singular pertrubation problems, Diff. Int. Eqns. 13(2000), 15-45.

[51] J. Wei, On single interior spike solutions of Gierer-Meinhardt system: uniqueness, spectrum estimates, Europ. J. Appl. Math. 10(1999), 353-378.

[52] J. Wei and M. Winter, Stationary solutions for the Cahn-Hilliard equation, Ann. Inst. H. Poincaré Anal. Non Linéaire, 15 (1998), 459-492.

[53] J. Wei and M. Winter, Multiple boundary spike solutions for a wide class of singular perturbation problems, J. London Math. Soc. 59 (1999), 585-606.

[54] J. Wei, Point-condensation generated by the Gierer-meinhardt system: a brief survey, book chapter in New Trend In Partial Differential Equations 2000, (Y. Morita, H. Ninomiya, E. Yanagida, and S. Yotsutani editors), pp. 46-59.

\section{Chang-Shou Lin}

Department of Mathematics, Chung Cheng University

Minghsiung, Chia Yi, Taiwan 
E-mail: cslin@math.ccu.edu.tw

Juncheng Wei

Department of Mathematics

The Chinese University of Hong Kong, Shatin, Hong Kong

E-mail: wei@math.cuhk.edu.hk 